\title{
La remise en question du pouvoir psychiatrique à Genève (1960-1980) ${ }^{1}$
}

\section{Federico Dotti}

\section{Résumé}

Cet article porte sur le développement des formes de remise en question du pouvoir psychiatrique dans le contexte genevois des années 1970, contestations qui s'inscrivent au sein d'un phénomène plus ample caractérisant la discipline psychiatrique depuis les années 1960 déjà et désigné par le terme d'«antipsychiatrie». Une présentation du contexte et des réformes concernant la Clinique de Bel-Air sera suivie d'une analyse des critiques et des revendications avancées par les différents collectifs composant la scène alternative en matière de psychiatrie: de la dénonciation de l'existence de la répression psychiatrique dans le Canton de Genève à l'éclatement de la crise et aux réorganisations des structures psychiatriques, jusqu'aux modifications législatives des années 1980.

Mots-clés: antipsychiatrie; pouvoir psychiatrique; collectifs alternatifs; droits des patients; Genève

\section{Summary}

The article deals with the development of the manner in which Geneva's institutional psychiatric power is undermined during the 1970s. An attempt is thus made to situate the former disagreements into a broader perspective

1 Cet article a été élaboré à la suite du travail de mémoire en vue de l'obtention de la maîtrise en science de l'éducation - éducation spéciale - à l'Université de Genève (2015): La remise en question de la psychiatrie: du mouvement de l'antipsychiatrie aux groupes militants dans la Genève des années 1970.

Federico Dotti, Équipe de didactique de l'histoire et de la citoyenneté (ÉDHICE), Faculté de psychologie et des sciences de l'éducation, Université de Genève, Pavillon Mail, 40, Boulevard du Pont d'Arve, CH-1211 Genève 4 (federico.dotti@unige.ch). 
thereby referring to the so-called «anti-psychiatry» of the 1960s. Following a presentation of the Bel-Air Clinic in regards to its context and reforms, the paper features an analysis of the criticisms and demands purported by alternative groups in matter of psychiatry. As such, the paper ranges from the denunciation of psychiatric repression in Canton Geneva, to the subsequent critical eruption of the reorganisation of the psychiatric structures, up to the legislative amendments of the 1980s.

Keywords: anti-psychiatry; psychiatric power; alternative groups; rights of patients; Geneva

\section{Introduction}

La remise en question du pouvoir psychiatrique à partir des années 1960, et plus particulièrement dans les années 1970, sur le territoire genevois, met en lumière des contradictions liées au domaine de la psychiatrie éveillant un certain intérêt parmi le personnel soignant et l'opinion publique. Ce phénomène s'inscrit dans une atmosphère de critique institutionnelle qui va se généralisant au niveau international et qui, pour ce qui concerne le domaine de la psychiatrie, est connue sous le terme d'«antipsychiatrie». Nonobstant une période de réformes importantes, marquée par une politique d'ouverture et le développement du secteur extrahospitalier, la Clinique de Bel-Air est confrontée à partir des années 1970 à des tensions internes et à la naissance d'une critique intra- et extra-muros. Celles-ci concernent le pouvoir psychiatrique et s'articulent en particulier autour de la question de la médicalisation des troubles psychiques et des droits des usagers de la psychiatrie, avec une forte composante sociale. De ce fait, ce questionnement interroge le rapport qu'entretiennent les autorités et la population avec la souffrance psychique et œuvre en faveur d'une plus grande tolérance. Cette période tumultueuse mène les institutions psychiatriques genevoises à la création d'une commission d'enquête par le Conseil d'État et au dévoilement consécutif dans l'espace public d'abus et de dysfonctionnements.

À partir d'ouvrages «antipsychiatriques», de sources militantes et engagées, tout comme d'articles de presse, complétés par quelques thèses de médecine, des documents des archives fédérales, une interview et des ouvrages historiographiques, cet article se propose de retracer la naissance, le développement et les revendications des collectifs ayant constitué la critique de l'institution psychiatrique dans le Canton de Genève. 


\section{Les années 1960 entre psychiatrie et antipsychiatrie}

Les années 1960-1970 sont marquées, sur les plans politique, culturel et symbolique par une mise en question de l'autorité, mais également par une critique des critères fixant les normes sociales, avec la revendication et la valorisation des différences interpersonnelles; des revendications qui s'inscrivent au sein des idéologies contre-culturelles se manifestant déjà à partir des années $1950 .{ }^{2}$ L'institution psychiatrique, extrêmement hiérarchisée et chargée d'établir la limite entre le «normal» et l'«anormal» en termes de comportement, n'échappe pas à ce mouvement culturel antiautoritaire et en deviendra même par la suite un objet privilégié, bien que la profondeur du questionnement autour de l'asile et de la folie demeure modérée et diverse d'un pays à l'autre autour de 1968. ${ }^{3}$ En particulier, dans les années 1960, différentes réflexions et expériences se développent sous des formes multiples et commencent à miner la stabilité de l'institution psychiatrique en contribuant à médiatiser un phénomène inédit, bientôt connu sous le nom d'«antipsychiatrie». ${ }^{4}$ Ce terme, mystifié, démystifié, exalté, rejeté, ${ }^{5}$ englobe une pluralité d'approches, ${ }^{6}$ dont le noyau central, source d'unité d'un mouvement hétérogène, est la remise en question du pouvoir psychiatrique: «ce supplément de pouvoir par lequel le réel est imposé à la folie au nom d'une vérité détenue une fois pour toutes par ce pouvoir sous le nom de science médicale, de psychiatrie». ${ }^{7}$ Or, ce mouvement ne prône pas l'abolition de la psychiatrie, mais des changements fondamentaux, voire une révolution dans le domaine. Cependant, bien que le terme d'antipsychiatrie s'accompagne d'une image de rupture nette avec la psychiatrie traditionnelle, ${ }^{8}$ l'institution asilaire et la profession de psychiatre faisaient déjà l'objet d'un débat dès la deuxième moitié du XIX ${ }^{\text {ème }}$ siècle. ${ }^{9}$ Ce questionnement s'intensifie progressivement, en particulier à partir des années de l'après-guerre, ${ }^{10}$ période pendant laquelle bon nombre d'hôpitaux psychiatriques font l'objet d'une politique réformiste. ${ }^{11} \mathrm{Ainsi}$, beaucoup des idées à la base du mouve-

2 Corbellini/Jervis 2008; Crossley 2006; Jones 1998.

3 Castel 1981; Coffin 2003; Foot 2014; Guattari 2012.

4 Ce terme parait pour la première fois en 1967 lors de la publication de l'ouvrage du psychiatre David Cooper Psychiatrie et Anti-psychiatrie (1967/1970).

5 Jervis 1977.

6 Foot 2014.

7 Foucault 2003, 132.

8 Gijswijt-Hofstra 1998; Jones 1998.

9 Voir notamment Brulhart 2002; Crossley 2006; Fauvel 2005; Nolte/Fangerau 2006; Quétel 2009.

10 Crossley 2006; Gijswijt-Hofstra 1998; Henckes 2007; Jones 1998; Thomson 1998.

11 Voir notamment Henckes 2007; Thifault/Dorvil 2014; Von Bueltzingsloewen 2007; Von Bueltzingsloewen 2013. 
ment antipsychiatrique ne sont pas tant des innovations en rupture avec le passé, comme on a pu les présenter à l'époque. Outre l'influence de la psychiatrie phénoménologique, ${ }^{12}$ certains concepts développés par des courants tels que celui de la psychiatrie sociale, ${ }^{13}$ ou de la communauté thérapeutique, ${ }^{14}$ avaient déjà modifié les pratiques asilaires, ce qui suggère des formes de continuité dans une réappropriation des questionnements précédant la critique étiquetée comme antipsychiatrique. Ceci aurait été un élément facilitateur de la diffusion et de la réception par le public des critiques de la psychiatrie radicale, comme le souligne Crossley. ${ }^{15}$ De ce fait, d'après Foucault, «l'ensemble de la psychiatrie moderne est au fond traversé par l'antipsychiatrie, si on entend par là tout ce qui remet en question le rôle du psychiatre chargé autrefois de produire la vérité de la maladie dans l'espace hospitalier». ${ }^{16}$ Cependant, poursuit l'auteur, le mouvement de «dépsychiatrisation» qui a accompagné le développement de la psychiatrie, dans un premier temps par la réduction de la maladie mentale aux seuls symptômes qui permettaient de la diagnostiquer et aux techniques nécessaires pour la soigner, et, dans un second temps, par le développement de la psychanalyse, maintient un rapport de domination du médecin sur le patient. Or, «à ces deux formes de dépsychiatrisation, toutes deux conservatrices du pouvoir, l'une, parce qu'elle annule la production de vérité, l'autre, parce qu'elle tente de rendre adéquats production de vérité et pouvoir médical, s'oppose l'antipsychiatrie». ${ }^{17}$

12 La psychiatrie phénoménologique s'oppose à la psychiatrie organiciste par son approche subjective qui conçoit la compréhension de l'état de «santé» ou de «folie» à partir du ressenti du sujet en relation avec le monde dans lequel il vit. Sur l'influence de cette approche au sein du mouvement de l'antipsychiatrie, voir notamment Galimberti 1979/2006.

13 Selon Thomson 1998, l'antipsychiatrie - en tout cas pour ce qui concerne la GrandeBretagne - s'inspire de la psychiatrie sociale de la Deuxième Guerre mondiale et de la période de reconstruction de l'après-guerre. Cette approche introduit des variables culturelles et sociales dans la compréhension des troubles psychiques.

14 La communauté thérapeutique, qui aura une forte influence sur les travaux de Basaglia, Cooper et Laing, est un modèle qui émerge en Grande-Bretagne dans le contexte de la Deuxième Guerre mondiale et qui se diffuse progressivement aux États-Unis et en Europe continentale, en particulier dans les années 1960, comme une alternative aux pratiques traditionnelles des hôpitaux psychiatriques. Cette approche se caractérise par la volonté d'affronter et de résoudre collectivement les problèmes propres aux institutions psychiatriques grâce à un système de réunions régulières auxquelles participent les professionnels, ainsi que les patients. La nouvelle attention portée sur les dynamiques interpersonnelles s'accompagne de ce fait d'une transformation de l'organisation hiérarchique. À ce sujet, voir notamment Fussinger 2010.

15 Crossley 2006.

16 Foucault 2003, 347.

17 Foucault 2003, 349. 
Ainsi, l'antipsychiatrie naît au sein de la psychiatrie. ${ }^{18}$ Parmi les expériences les plus connues auxquelles est attribué un caractère antipsychiatrique se trouvent notamment l'œuvre de désinstitutionalisation de Franco Basaglia en Italie commencée à l'hôpital de Gorizia, ${ }^{19}$ le Pavillon 21 dirigé par David Cooper, ${ }^{20}$ ou encore Kingsley Hall, projet animé par Ronald Laing auquel collabore également David Cooper. ${ }^{21}$ Basaglia souligne que la puissance de ces expériences réside dans la démonstration que l'impossible devient possible. ${ }^{22}$ Autre représentant du mouvement de l'antipsychiatrie, le nord-américain Thomas Szasz est célèbre pour sa pensée très radicale qui n’a cependant pas connu de transposition en expérience pratique. ${ }^{23}$ Or, si l'identification au terme d'antipsychiatre n'est pas revendiquée, mais souvent attribuée et reniée, ${ }^{24}$ le courant idéologique qui est à la base du mouvement de l'antipsychiatrie se révèle distinctement reconnaissable. ${ }^{25}$ L'antipsychiatrie considère la psychiatrie comme faisant partie du problème, ${ }^{26}$ et c'est bien cet aspect essentiel du questionnement qui confère à ce courant un caractère distinct de toute autre mise en question ayant accompagné de tout temps la discipline psychiatrique. ${ }^{27}$ Face au monopole du savoir médical, on assiste à la valorisation des «savoirs assujettis», ${ }^{28}$ soit ceux du malade, du psychiatrisé, mais aussi de l'infirmier, qui, disqualifiés, côtoient depuis toujours le savoir médical d'une façon marginale. ${ }^{29}$ De ce fait, comme l'explique Cooper, «une mise en question plus radicale a conduit certains d'entre nous [travailleurs dans le champ de la psychiatrie] à proposer des conceptions et des procédures qui semblent s'opposer absolument aux conceptions et procédures traditionnelles - et qui, en fait, peuvent être considérées comme le germe d'une anti-psychiatrie». ${ }^{30}$

Ce phénomène semble trouver un terrain favorable à son éclosion dans la situation de crise réelle que connaît la psychiatrie institutionnelle, ${ }^{31}$ mais pas seulement. Si beaucoup de questionnements à la base du mouvement étaient

18 Foot 2014.

19 Voir Basaglia 1967; Basaglia 1968/2012a-b; Basaglia/Basaglia-Ongaro 1971/2013.

20 Voir Cooper 1967/1970; Cooper 1971/1972.

21 Voir Laing 1960/1970; Laing 1961/1971; Laing 1969/1979; Barnes/Berke 1971/1973; Boyers 1971/1973.

22 Basaglia 2000; Coffin, 2008.

23 Voir Szasz 1961/1975; Szasz 1970/1976; Szasz 1963/1977.

24 Foot 2014; Guattari 2012; Jervis 1977.

25 Corbellini/Jervis 2008; Jervis 1977.

26 Coffin 2008; Foot 2014; Jones 1998.

27 Foucault 2003.

28 Foucault 1997, 8.

29 Ibid.

30 Cooper 1967/1970, 7.

31 Basaglia 1968/2012b; Cooper 1967/1970; Corbellini/Jervis 2008; Jervis 1968/2012. 
déjà présents au sein de la communauté psychiatrique avant l'apparition du terme antipsychiatrie, ce qui caractérise ce mouvement ne semble pas tant être la nature du discours que la manière de le formuler, ainsi que la réceptivité du publique auquel s'adresse tel discours: ${ }^{32}$ l'antipsychiatrie est «d'abord un phénomène littéraire, mass-médiatique», ${ }^{33}$ «un nom que les consommateurs de la culture et de la mode ont attribué tour à tour à des courants différents de la psychiatrie et à des psychiatres particuliers». ${ }^{34}$ Or, comme toute étiquette, le terme d'antipsychiatrie tend à simplifier et à réduire une multitude de caractères par une sélection d'idées qui permet d'en délimiter le contenu. ${ }^{35}$ L'antipsychiatrie représente ainsi un système d'opposition avec un modèle simplifié de psychiatrie traditionnelle, souligne Jones. ${ }^{36}$

La propagation des idées prônées par l'antipsychiatrie dépasse le cadre médical et professionnel, forte de l'intérêt croissant de l'opinion publique pour les questions du domaine de la psychiatrie qui se manifeste particulièrement dans les milieux des cultures alternatives et underground propres à la fin des années 1960 et début des années 1970, ${ }^{37}$ dont l'antipsychiatrie ira même jusqu'en devenir un vecteur. ${ }^{38}$ Sur ce sujet, Coffin relève que ces tentatives concrètes de rupture avec le milieu psychiatrique traditionnel ravivent la braise du mouvement de Mai 1968 en étant porteuses d'un imaginaire de liberté et en se prêtant à une lecture de type militant. ${ }^{39}$ D'une certaine manière, le succès médiatique de l'antipsychiatrie dépend de la capacité du mouvement et de ses représentants de répondre aux attentes d'une vague de rébellion qui caractérisait l'ère du temps ${ }^{40}$ «la contestation anti-psychiatrique a été ainsi un point de fixation privilégié d'un imaginaire politique de la libération vécu à l'époque sous la forme d'une sensibilisation exacerbée à la répression» ${ }^{41}$ Cependant, si, d'après Barham, la critique de l'antipsychiatrie, ou psychiatrie radicale, a été plus significative dans le contexte de l'histoire culturelle que dans celui de la psychiatrie, ${ }^{42}$ Crossley souligne que ce mouvement a inspiré beaucoup d'acteurs du domaine et de patients à agir différemment. ${ }^{43}$

32 Crossley 2006; Gijswijt-Hofstra 1998; Jones 2008.

33 Guattari 2012, 257.

34 Jervis 1977, 32.

35 Crossley 2006.

36 Jones 1998.

37 Crossley 2006; Gijswijt-Hofstra 1998; Guattari 2012; Jones 1998.

38 Guattari 2012; Ingleby 1998; Thomson 1998.

39 Coffin 2008.

40 Crossley 2006.

41 Castel 1981, 23.

42 Barham 1998.

43 Crossley 2006. 
Le point de départ de cette remise en question est l'hôpital psychiatrique: lieu à vocation thérapeutique, cette institution totale se caractérise par son haut niveau d'exclusion et de contrôle. Selon cette vision critique, la personne internée et investie du statut de malade est exclue du jeu social et se trouve à la merci du pouvoir psychiatrique. Elle est comprise au travers d'un système technico-psychiatrique dont la seule méthode explicative relève de la maladie mentale. ${ }^{44}$ À ce propos, Basaglia souligne la misère des conditions d'hospitalisation du malade dans l'asile: ${ }^{45}$ «un homme sans droits». ${ }^{46}$ Ainsi, d'après Coffin, le mouvement de l'antipsychiatrie se transforme à partir des années 1970 et dépasse la critique des pratiques psychiatriques pour élargir la contestation aux institutions, aux hiérarchies et à l'organisation du savoir. ${ }^{47}$ Le mouvement fait brèche tant auprès du grand public que chez les professionnels du domaine de la psychiatrie en dénonçant l'existence de la répression dans le domaine psychiatrique: une relation entre cette forme de répression et les autres est alors dévoilée..$^{48}$ Cela met à jour une connivence entre l'institution psychiatrique et le système judiciaire en termes de contrôle social et de contention des phénomènes déviants. ${ }^{49}$ De la sorte, comme le souligne Basaglia, cette critique ne se limite pas au seul domaine médicopsychiatrique:

La polémique engagée contre le système institutionnel dépasse le domaine de la psychiatrie, pour atteindre les structures sociales qui les sous-tendent; en nous obligeant à remettre en cause la neutralité scientifique - inféodée aux valeurs dominantes - elle se mue en critique et en action politique..$^{50}$

De ce fait, ce mouvement comporte un caractère complexe qui unit des formes de critique et d'autocritique sociale, culturelle et politique (anti-autoritaire et anti-institutionnelle) de nature diverse. ${ }^{51} \mathrm{La}$ remise en question se développe transversalement touchant l'institution psychiatrique dans sa globalité: de la fonction du psychiatre au mandat social de la psychiatrie, de la visée thérapeutique des structures hospitalières à leur système hiérarchique, ${ }^{52}$ allant même jusqu'à interroger l'essence de la maladie mentale,

44 Goffman 1961/2013. L'analyse sociologique développée dans l'ouvrage Asiles, études sur la condition sociale des malades mentaux de Erving Goffman aura une grande influence sur le développement de la critique psychiatrique des années 1960-1970, tout comme l'Histoire de la folie à l'âge classique de Michel Foucault.

45 Basaglia 2000.

46 Basaglia 1968/2012, 132.

47 Coffin 2008.

48 Guattari 2012.

49 Basaglia et Basaglia-Ongaro 1971/2013.

50 Basaglia 1968/2012a, 21.

51 Castel 1981; Coffin 2008; Foot 2014.

52 Voir notamment Basaglia 1968/2012b; Goffman 1961/2013. 
source de légitimité d'une psychiatrie qui se veut une discipline médicale et scientifique. ${ }^{53}$ En particulier, d'après Foot, ce dernier point a contribué au développement d'une conception antihistorique et simpliste qui a fini par imposer l'idée que l'antipsychiatrie se résume à la négation de la maladie mentale. Or, si cette prise de position est fortement défendue par Szasz, il n'en va pas de même de Laing qui comprend les comportements liés à la schizophrénie comme une réponse à des relations interpersonnelles dans le milieu familial considérées comme hostiles, et encore moins de Basaglia pour qui ces comportements relèvent de facteurs sociaux dans un espace élargi au-delà du cercle familial et qui propose une approche caractérisée par une mise en parenthèse de la maladie mentale. ${ }^{54}$

Le mouvement de l'antipsychiatrie soulève, au travers de questionnements inhérents à la médicalisation, à la répression et à l'exclusion du fou, des réflexions plus profondes qui touchent à l'organisation des pouvoirs politiques en termes de prise en charge d'une partie de la population dont la souffrance côtoie le stigmate de la déviance. Ainsi, d'après Cooper, «l'anti-psychiatrie était et demeure la lutte au sein des institutions publiques d'hôpitaux et de secteurs, visant à détruire ce système de l'intérieur».55

\section{Genève: Ajuriaguerra, la politique réformiste et ses limites}

Lorsque des expériences alternatives voient le jour, l'institution psychiatrique genevoise n'opère pas de changements radicaux, préférant une action réformiste à une politique de transformation..$^{56}$ Sous l'impulsion du directeur Julián De Ajuriaguerra, entré en fonction le $1^{\text {er }}$ avril 1959, on assiste à une réorganisation interne de la Clinique de Bel-Air et à une tentative du développement progressif de l'activité extrahospitalière. ${ }^{57}$ Ainsi, un nouveau rapport entre la psychiatrie et la communauté se met en place avec la volonté de sortir de la condition d'isolement qui caractérise la structure hospitalière inaugurée en 1900. ${ }^{58}$ Lintroduction en 1953 des médicaments psychotropes à la Clinique de Bel-Air a sans doute contribué, comme partout ailleurs, à cette conception renouvelée de la psychiatrie et de l'hôpital psychiatrique. En effet, d'après Bovier, la thérapie médicamenteuse vient se substituer à la

53 Voir notamment Canguilhem 1966/2013; Foucault 1954/2011; Foucault 1972/2013; Foucault 2003; Szasz 1961/1975.

54 Coffin 2008; Foot 2014.

55 Cooper $1977,40$.

56 Bimpage 1981, 27 octobre.

57 Bovier 1981; Brulhart 2003.

58 Brulhart 2003; Joanes-Smidof 1984. 
contention physique. En diminuant l'agitation, elle permet de ce fait d'instaurer un nouveau rapport entre le personnel et le patient; elle favorise l'ouverture de la Clinique vers l'extérieur et contribue même à jeter les bases de la critique de l'institution psychiatrique. ${ }^{59}$ Sur ce sujet, Alain Riesen affirme:

$\mathrm{Au}$ moment où il y a l'arrivée des neuroleptiques comme nouveau médicament, on peut enlever les barreaux dans le dispositif institutionnel, on peut considérer les professionnels comme des soignants et pas comme des gardiens de «prison», on peut éliminer les traitements de choc progressivement, etc. Un élément de ce dispositif [institutionnel], lorsqu'il change de manière significative, il change aussi les autres, ils sont interconnectés. ${ }^{60}$

À partir de 1960, la vie sociale des patients devient le pivot autour duquel s'articule la période de réorganisations structurales qui vise à reproduire à l'interne des conditions de vie aussi proches que possible de celles de la vie à l'extérieur de la Clinique. C'est ainsi le début de la rénovation intérieure des pavillons et de leur ouverture afin d'en supprimer l'aspect carcéral, la construction de nouvelles unités, mais également de l'aménagement d'ateliers pour l'ergothérapie qui est pratiquée de manière systématique dans chaque pavillon à partir de 1966. En outre, l'introduction et le développement de la sociothérapie au niveau pavillonnaire et institutionnel, ainsi que la mise en place des psychothérapies pavillonnaires, donnent à voir une orientation thérapeutique combinant deux axes: médico-biologique et social. L'évolution souhaitée concerne également le personnel médical et infirmier au niveau de la formation, des effectifs et de l'organisation du travail. Cette approche vise à redéfinir l'hôpital comme lieu de soins et à éviter l'isolement social des personnes hospitalisées. Elle souligne l'importance de la relation et du contact humain dans une structure qui doit fonctionner comme une communauté thérapeutique. ${ }^{61}$ À ce propos, Ajuriaguerra souligne que

ce qui compte, c'est l'utilisation fonctionnelle de l'instrument hospitalier considéré comme une communauté thérapeutique, susceptible de permettre le maximum de soins dans un cadre humain. En outre, l'hôpital [...] fait partie de l'équipement sanitaire d'une population, et [...] doit être inséré dans un plan général où il est l'intermédiaire entre des organismes préventifs, des organismes de reclassement médico-social et des services d'hospitalisation de longue durée. ${ }^{62}$

59 Bovier 1981.

60 La lutte contre les abus de la psychiatrie. Entretien avec M. Alain Riesen. Alain Riesen est ergothérapeute de formation. Il a travaillé à la Clinique de Bel-Air et a participé à la création du Comité d'Action «Santé» (CAS) en 1972, du Réseau Suisse Romand d'alternative à la psychiatrie en 1976 et de l'association pour les droits des usagers de la psychiatrie (ADUPSY) en 1979. En 1984, il a contribué à la fondation de l'Arcade 84 qui est un centre d'ergothérapie et un centre de jour pour des personnes en souffrance psychique. Il a également participé à la création de l'association Pro Mente Sana en 1992.

61 Bovier 1981; Brulhart 2003.

62 Ajuriaguerra cité par Bovier 1981, p. 92. 
Cette humanisation de l'hôpital s'oriente ainsi vers une diminution des contraintes, une majeure participation des patients et du personnel à l'organisation de la vie dans les pavillons et l'offre de loisirs et du travail selon les désirs et les possibilités. ${ }^{63}$ Cependant, cette mutation de la Clinique en lieu de soins demande du temps. En effet, Riesen décrit ainsi son expérience de travail en tant qu'aide-soignant dans un pavillon fermé de la Clinique de Bel-Air en 1967:

J'ai vu ce qu'on appelle la violence institutionnelle, c'est à dire un ensemble de patients dans une salle, à peu près deux fois comme celle-ci [ $4 \times 2 \mathrm{~m}$.], nue, avec deux tables fixées au sol, deux WC ouverts sans portes et puis des hommes mélangés: troubles psychotiques, handicaps physiques en chaise roulante, handicaps mentaux enfin un mélange. Ils passaient la journée dans cette salle et il y avait toujours un aide-soignant ou un infirmier qui était là en train de les surveiller. On leur donnait à manger à midi, une espèce de porridge, purée... Ils mangeaient vers 16.30, 17.00 heure et après on les couchait. Il y en a qui étaient attachés sur leurs lit, soi-disant qu'ils faisaient leurs urines, et ils étalaient leur excréments sur les murs, alors on les attachait. Ils restaient attachés toute la nuit. ${ }^{64}$

Parallèlement à la réorganisation interne, la psychiatrie institutionnelle développe un projet de soins extrahospitaliers dont la création du Centre Psychosocial Universitaire (CPSU) en 1963 sera la première étape d'un système dit de sectorisation. Cette réforme se poursuivra avec la répartition de la Clinique de Bel-Air par secteurs géographiques et la multiplication d'équipes médico-sociales, de centres de consultations et des foyers dans la ville, en particulier au cours des années 1970; mais aussi avec des centres de thérapies brèves au cours des années 1980, l'idée étant de promouvoir une ouverture de la psychiatrie vers la cité. ${ }^{65}$ En effet, en 1974, la Clinique est réorganisée et divisée en trois secteurs qui correspondent à l'extrahospitalier. Les pavillons ouverts sont ainsi attribués chacun à un secteur géographique: Les Tilleuls (désormais mixte) pour le secteur de Pâquis, Les Lilas Blancs (femmes) et Le Salève (hommes) pour le secteur de la Jonction, Les Voirons (femmes) et La Seymaz (hommes) pour le secteur des Eaux-Vives. Si, jusque-là, le partage de la gestion de la Clinique s'établissait entre division-hommes et division-femmes, gérées chacune par un médecin-chef, à présent chaque secteur est confié à un médecin-chef. Cette étape est censée jeter les bases en vue d'une sectorisation complète de la Clinique prévue pour 1976-1977, où chaque secteur inclura une unité d'admission, celles-ci demeurant encore pour l'instant sous la responsabilité d'un quatrième médecin-chef puisqu'elles ont conservé un caractère d'unité spécialisée. ${ }^{66} \mathrm{Un}$ autre facteur fondamental du projet est la

63 Bovier 1981; Joanes-Smidof 1984.

$64 \mathrm{La}$ lutte contre les abus de la psychiatrie. Entretien avec M. Alain Riesen.

65 Bovier 1981; Brulhart 2003; Droze 2003.

66 Bovier 1981; Brulhart 2003. 
réinsertion par le travail avec l'ouverture de plusieurs ateliers en collaboration avec l'Assurance Invalidité. ${ }^{67}$

Ajuriaguerra souligne que pour ouvrir l'hôpital psychiatrique il est nécessaire de développer le secteur extrahospitalier, tout comme de refuser que 700 personnes soient enfermées sur la base du risque potentiel d'une dangerosité encore associée à l'idée du fou, du fou furieux. ${ }^{68}$ À ce sujet, Droze relève que l'ouverture de l'institution psychiatrique vers la cité aurait permis de diminuer le poids du stigmate de la maladie mentale. Cette politique de secteur est également soutenue par les conseillers d'État chargés de la santé, Jean Treina, de 1963 à 1965, puis Willy Donzé. ${ }^{69}$

Après une période d'enthousiasme et de grands changements, la politique réformiste introduite par Ajuriaguerra entre en crise à partir de 1969.70 La conception du «village de Bel-Air» s'essouffle, les idées novatrices s'estompent, les résistances internes prennent davantage de poids, ${ }^{71}$ et la Clinique est confrontée au contexte international et à un air du temps de critique institutionnelle qui prend de l'ampleur. ${ }^{72}$ Ceci est confirmé par Riesen qui souligne qu'il y a

\begin{abstract}
un moment de contestation extrêmement puissant des pouvoirs constitués, des pouvoirs institutionnels, que ça soit la famille, l'usine, la santé, l'éducation, etc. Il y a un mouvement de contestation des normes institutionnelles, ça c'est le mouvement global. Nous, comme jeunes étudiants et professionnels, on est dans cette dynamique-là, on est dans une dynamique de réflexion, de contestation des pouvoirs institués. ${ }^{73}$
\end{abstract}

À ce sujet, nous pouvons souligner que les idées antipsychiatriques se propagent dans le Canton de Genève à partir du début des années $1970,{ }^{74}$ et que le climat de critique institutionnelle se reflète dans la naissance d'un certain nombre de groupes et mouvements qui proposent des réflexions autour des diverses contradictions qui hantent toujours le domaine de la psychiatrie genevoise. ${ }^{75}$

67 Droze 2003.

68 Julian de Ajuriaguerra Interview lors de l'émission en direct avec du 4 octobre 1971.

69 Droze 2003.

70 Brulhart 2003.

71 Bovier 1981.

72 Brulhart 2003.

73 La lutte contre les abus de la psychiatrie. Entretien avec M. Alain Riesen.

74 Bimpage 1981, 27 octobre. La médiatisation des idées et des expériences reliées au mouvement de l'antipsychiatrie semble se confirmer par la présence de nombreux articles publiés au cours des années 1970-1980 dans Le Journal de Genève et La Gazette de Lausanne (dont les archives sont numérisées: www.letempsarchives.ch). À ce sujet, voir aussi Rebetez 2014 qui aborde l'antipsychiatrie dans l'Arc lémanique d'après quatre groupes d'acteurs: psychiatres, soignants, personnes psychiatrisées et journalistes.

75 Ces collectifs s'incrivent dans la scène alternative genevoise qui connaît un développement remarquable à partir des années 1970 avec la création de plus de 200 groupements et comités d'action alternative. À ce sujet, voir notamment Gros 1987; Gros 2004. 
Ainsi, les premières réflexions se concrétisent en 1972 avec la naissance du Comité d'Action «Santé» (CAS). Composé majoritairement de travailleurs des institutions sanitaires et des étudiants, ce groupe se développe autour de l'hôpital. Il propose une réflexion transversale concernant tout le champ de la médecine et préconise de tisser des liens avec d'autres formes de luttes sociales présentes dans la ville. ${ }^{76}$ D'après Riesen, membre fondateur, le CAS s'intéresse à la réalité institutionnelle en intégrant sa dimension politique. ${ }^{77}$

En 1973, le groupe Travail Social et Psychiatrie voit le jour. Ses membres se réunissent dans le but de prendre connaissance des expériences de psychiatrie innovante ou d'antipsychiatrie dans d'autres contextes et de proposer des analyses des différentes institutions psychiatriques présentes sur le territoire genevois. Ils affirment qu'à Bel-Air, la distribution du pouvoir reste fortement hiérarchisée, ce qui impose des cadres très stricts et limite notablement la recherche de nouvelles formes de prise en charge ne s'inscrivant pas dans la ligne de conduite institutionnelle. ${ }^{78}$ La même année, l'existence de la violence institutionnelle est médiatisée par la parution d'un dossier concernant la psychiatrie dans le mensuel Tout Va Bien, dossier élaboré par des travailleurs du domaine de la psychiatrie, des usagers, des syndicalistes, ainsi que des journalistes. On y dénonce notamment la prédominance de l'approche médicamenteuse et le manque de préparation à la sortie, l'invalidation de la parole du malade et le secret médical comme responsables de la ségrégation des usagers de la Clinique de Bel-Air, mais également la structure pyramidale du pouvoir et les divisions hiérarchiques: «soumission de l'infirmier au pouvoir médical et soumission souvent du médecin aux pouvoirs de l'administrateur bailleur de fonds». ${ }^{79}$

De l'intérieur, les contestations au sein de l'institution psychiatrique se poursuivent; ainsi, en 1974, une lettre est adressée à la direction de la Clinique de Bel-Air par l'Assemblée des médecins-assistants et des chefs de clinique. Ces derniers affirment leur volonté de s'abstenir de la recherche psychopharmacologique en raison d'un manque de clarté concernant la méthodologie des investigations. ${ }^{80}$

La présence de ces premières critiques sur le territoire genevois met en évidence la primauté de l'approche médicale dans l'institution. En effet, bien que l'utilisation des neuroleptiques aient permis une nouvelle conception des soins en psychiatrie, cette approche semble s'être imposée au détriment

76 CAS 1. Journal du Comité d'Action «Santé». Mai 1972.

77 La lutte contre les abus de la psychiatrie. Entretien avec M. Alain Riesen.

78 Travail social et Psychiatrie. Séance du lundi 5 novembre 1973.

79 Tout Va Bien. LE PREAU DES FOUS. Eté 73, 14.

80 L'assemblée des médecins-assistants et chefs de clinique de la Clinique Psychiatrique de Bel-Air. Genève, le 4 juin 1974. 
d'une dimension sociale qui reste marginale. Cette conception des soins se révèle comme la source de légitimation du pouvoir du psychiatre et, par conséquent, d'une gestion structurale fortement hiérarchisée. Or, comme le relève Brulhart, les conflits sociaux qui perturbent l'institution jusqu'au départ du directeur Ajuriaguerra en 1976 ne sont que des préludes à de plus profonds changements ultérieurs. ${ }^{81}$

\section{Le changement de direction et le développement de la critique institutionnelle}

\section{La critique du Secteur}

En 1975, une délégation suisse romande participe à une rencontre à Bruxelles, rencontre qui voit naître le Réseau International «Alternative à la Psychiatrie». D'après Guattari, ce Réseau se veut une réponse à la nécessité de dépasser les formes de lutte locales qui ont certes permis de montrer qu'une autre manière de faire de la psychiatrie était possible, mais qui n'ont pas eu la force de s'imposer de manière durable face à la puissance de l'institution psychiatrique traditionnelle. Le thème de la rencontre est «l'alternative au Secteur». Parmi les participants se trouvent des professionnels ayant participé à la modernisation de la psychiatrie française et au développement de la politique du Secteur et désormais confrontés aux limites de celle-ci. ${ }^{82}$ Le pouvoir psychiatrique reste le noyau central alimentant les critiques et les revendications de ce nouveau collectif. En effet, comme le souligne Elkaïm, les membres du Réseau International «Alternative à la Psychiatrie» visent la

suppression de toutes les formes d'enfermement psychiatrique, refus du monopole des professionnels sur les problèmes de la santé mentale, critique du secteur comme mise en place d'une relève technocratique à l'asile, soutien aux luttes menées par des groupes ou la population des quartiers pour prendre en main leur propre existence et éviter la psychiatrisation de la vie tout entière, critiques des techniques qu'elles soient psychiatriques ou psychanalytiques, etc. ${ }^{83}$

L'engagement des romands se concrétise une fois de plus avec la naissance du Réseau suisse-romand d'Alternative à la Psychiatrie (1976), dont le siège est établi à Genève. S'inscrivant dans un cadre aussi bien local qu'international, le travail de ce Réseau s'articule entre autre autour de la critique de la secto-

81 Brulhart 2003.

82 Guattari 2012.

83 Elkaïm 1977, 10. 
risation, de l'étude des nouveaux projets de loi et des droits des internés. ${ }^{84}$ Le caractère politico-social de sa critique semble s'inscrire dans la lignée d'un dépassement de la simple mise en question des pratiques psychiatriques telle qu'elle est revendiquée par les représentants du mouvement de l'antipsychiatrie. Au niveau international, tout comme dans le Canton de Genève, la critique psychiatrique investit toute la complexité des ramifications de l'institution qu'elle vise, y compris sa dimension juridique.

Nonobstant les réformes, la critique institutionnelle n'épargne pas l'institution psychiatrique genevoise qui est de surcroit confrontée à une période de rivalités et de luttes de pouvoir internes suite au départ d'Ajuriaguerra. En effet, cet événement marque la fin de la direction médicale unique, celle du Professeur Morel et du Professeur Ajuriaguerra. Ainsi, le $1^{\text {er }}$ octobre 1976, la commission administrative confie la direction des Instituts Universitaires de Psychiatrie de Genève (IUPG) au Dr Gaston Garrone et la direction de la Clinique de Bel-Air au Dr René Tissot. ${ }^{85}$ Comme le souligne Brulhart, cette direction bicéphale se révèlera nuisible et sera responsable du ralentissement du développement de la sectorisation. ${ }^{86}$

Or, la restructuration de l'institution psychiatrique genevoise aurait dû permettre de résoudre dans une certaine mesure les contradictions caractérisant l'hôpital psychiatrique. Cependant, d'après les membres du Réseau suisse-romand d'Alternative à la Psychiatrie, si l'institution se trouve désormais modernisée, l'hôpital reste toujours la colonne portante de l'appareil psychiatrique: «la réforme de la psychiatrie à Genève, au travers de la sectorisation, consolide les murs de l'asile, ou ces fenêtres sans barreaux». ${ }^{87}$ Guattari, membre du Réseau International «Alternative à la Psychiatrie», considère que les innovations techniques et la sectorisation transforment certes l'image de l'hôpital psychiatrique, mais en reproduisent la même logique répressive qui demeure imprégnée de l'idéologie médicale. Ainsi, l'objectif devrait être la «dépsychiatrisation de la folie».88 À ce propos, Bierens de Haan souligne combien cette idéologie, ancrée à la tradition de l'hôpital général, entretient «cette idée préconçue, gratuite et nocive, que le lieu de soins psychiatriques doit ressembler au lieu de soins somatiques». ${ }^{89}$

84 Réseau Suisse-Romand «Alternative à la Psychiatrie», La guerre des psychiatres aura-t-elle lieu? Genève, juillet 1977, bulletin $\mathrm{n}^{\circ} 3$.

85 Rapport de la commission d'enquête sur les institutions universitaires psychiatriques genevoises, du $1^{\text {er }}$ septembre 1981. Séance du 8 octobre 1981 (après-midi).

86 Brulhart 2003.

87 Réseau suisse-romand «alternative à la psychiatrie». Genève, mai 1976, bulletin nº 2, 32 .

88 Guattari 2012, 266.

89 La communauté thérapeutique dans l'hôpital psychiatrique, courant d'espoir pour soignés et soignants. B. Bierens de Haan, 1. Barthold Bierens de Haan est psychiatre-psychothérapeute de formation. Il a travaillé à la Clinique de Bel-Air où il a été notamment animateur 
Mais qu'est-ce qu'on entend par dépsychiatrisation de la folie? Et de quelle manière pourrait-on envisager celle-ci? Selon Riesen, mettre fin au monopole de la psychiatrie sur la folie, à cette médicalisation de la déviance, nécessiterait d'inverser les priorités au sein des politiques en matière de psychiatrie sur le territoire genevois en mettant l'accent sur une réelle politique orientée sur les soins et l'intégration plutôt que d'axer le débat sur la sauvegarde de l'ordre public. ${ }^{90}$

Porte-parole du malaise croissant au sein de la Clinique psychiatrique genevoise, le Réseau Bel-Air est créé en 1976 grâce à une partie des soignants qui se battent pour une amélioration des conditions hospitalières, tant pour les personnes hospitalisées que pour les travailleurs. Les principaux points qu'il soulève concernent notamment la privation des droits que subissent les patients en franchissant les portes de la Clinique, la prédominance du modèle médical en dépit de la situation sociale du patient et l'excessive hiérarchisation de la structure. ${ }^{91}$ Ce mécontentement semble en autre coïncider avec la phase d'essoufflement de la politique hospitalière réformiste d'Ajuriaguerra, ${ }^{92}$ mais est également alimenté par trois affaires d'internement administratif dont nous parlerons par la suite.

Dans les mêmes années, deux médecins promeuvent une expérience de communauté thérapeutique. Or, bien que Tissot considère important que la communauté thérapeutique puisse s'aventurer dans la responsabilisation des patients et la libéralisation de la vie pavillonnaire, ceci doit être en accord avec les principes de l'institution. La communauté thérapeutique ne doit donc en aucun cas contrevenir à l'éthique conventionnelle telle qu'elle est défendue au sein de la Clinique de Bel-Air. ${ }^{93}$ Cet essai est par conséquent toléré, mais avec scepticisme. Il se veut une tentative de fournir une approche de la souffrance différente de celle proposée au sein de la Clinique psychiatrique genevoise que Bierens de Haan, l'un des deux médecins, n'hésite pas à qualifier d'«antithérapie».94 En effet, d'après ce dernier, l'institution psychiatrique n'as pas connu de véritable changements et le modèle médical

d'une communauté thérapeutique dans les années 1970. Il a été chef de clinique à l'hôpital psychiatrique universitaire de Genève et s'est intéressé aux thérapies par le milieu. En 1990, il s'est engagé auprès du Comité international de la croix rouge. Il est notamment l'auteur du Dictionnaire critique de Psychiatrie et de La Nostalgie de la Folie. Il exerce aujourd'hui en tant que médecin psychiatre et psychothérapeute à Paris.

90 La naissance de l'institution pour «aliénés» à Genève au XIX ${ }^{\mathrm{e}}$ siècle et la problématique de l'internement. Par Alain Riesen.

91 Réseau suisse-romand «alternative à la psychiatrie». Genève, mai 1976, bulletin nº 2 .

92 Bovier 1981.

93 Journal de Genève 1977, 27 septembre.

94 La communauté thérapeutique dans l'hôpital psychiatrique, courant d'espoir pour soignés et soignants. B. Bierens de Haan, 2. 
des troubles psychiques reste le modèle de référence, monopolisant les actes thérapeutiques (traitements pharmacologiques, électrochocs, cures de sommeil, isolement) enfermant le patient dans le rôle de malade. ${ }^{95}$

De ce fait, il semblerait que les autres approches comme la psychanalyse, l'ergothérapie et la sociothérapie, introduites durant la période réformiste par Ajuriaguerra, n'aient pas véritablement modifié l'orientation de l'institution qui reste centrée sur le modèle médical. En outre, la distribution fortement pyramidale du pouvoir caractérise encore l'institution, ce que dénoncent les groupes et mouvements critiques. Ceci semble confirmé par le licenciement des deux médecins en juillet 1977 suite à une divergence d'opinion avec le directeur Tissot concernant un traitement aux électrochocs appliqué contre son gré à une militante antinucléaire (Anna B.). Les mobilisations et les manifestations de solidarité envers les deux médecins sont nombreuses. ${ }^{96}$ Cet événement médiatise une réalité conflictuelle au sein de la Clinique de Bel-Air où subsistent des désaccords en termes d'action thérapeutique et de politique de soin. ${ }^{97}$ Les groupes syndicaux de la Clinique - la VPOD et le syndicat chrétien -, ainsi que les travailleurs et travailleuses psychiatriques dénoncent dans une lettre ouverte publiée dans le Journal de Genève l'arbitraire et le caractère ambigu de cette mesure qui se présente comme une (ré)-légitimation «d'une politique d'ordre et de pouvoir au détriment d'une politique de dialogue et d'ouverture». ${ }^{98}$

Plus de dix ans après la naissance des premiers mouvements de remise en question, la critique du pouvoir psychiatrique reste le noyau dur de la lutte autour de l'institution psychiatrique dans ses liens avec le système politique, social et juridique. Selon les membres du Réseau suisse-romand d'Alternative à la Psychiatrie, avec une distribution du pouvoir très hiérarchisée, la psychiatrie est une institution difficilement attaquable et, à Genève, contrairement à ce qui a pu se produire en Italie ou en Angleterre, aucun membre de ces groupes n'a l'autorité nécessaire lui permettant d'imposer de tels changements. De la sorte, les travailleurs sociaux et psychiatriques convergent dans leurs efforts de médiatisation des luttes afin de sensibiliser et d'impliquer la population, combinant de ce fait une critique interne à une critique extra-muros. ${ }^{99}$ Ainsi, en 1978, le groupe Bel-Air du syndicat VPOD publie Info-Bel-Air, bulletin qui s'intéresse aux difficultés propres à la structure psychiatrique genevoise, mais également à des problèmes de société et à 95 Ibid.

96 Voir Heimberg 2005.

97 Jullien 1977, 25 juin.

98 Journal de Genève 1977, 28 juillet, 11.

99 Réseau suisse-romand «alternative à la psychiatrie». Trieste, la fin d'un hôpital psychiatrique! Genève, novembre, 1977 , bulletin $\mathrm{n}^{\circ} 4$. 
d'autres formes de lutte. En particulier, le Groupe de travail sur la psychiatrie concentre ses réflexions autour des soins, de la relation entre professionnels et patients, ainsi que sur la formation et la communication au sein de l'équipe. ${ }^{100}$

La politique réformiste qui a conduit à la création du secteur est pointée du doigt et jugée en échec par la prédominance de l'approche médicale qui continue à caractériser l'idéologie de l'institution et qui limite le développement d'autres formes de prise en charge. La solution serait-elle la fermeture de l'hôpital? Quelles seraient les conséquences d'un acte si radical? Selon les membres du Réseau suisse-romand d'Alternative à la Psychiatrie,

une fois la fermeture de l'asile réalisée, l'expérience trouvera son espace ou ses limites dans les contradictions sociales elles-mêmes. Autrement dit: soit les forces sociales sont capables d'accepter une confrontation à la folie et de construire les solutions particulières à chaque aspect de ces contradictions (au niveau du quartier, de l'immeuble, du lieu de travail, etc.,); soit, dans le cas contraire, il se mettra en place un nouveau système de contrôle proche de ce que nous connaissons en Suisse dans le secteur, dont le développement peut s'appuyer sur une stabilisation du pouvoir socio-politique. [... $]^{101}$

Psychiatrisation de la population et répression psychiatrique

Entre 1937 et 1964 le total des personnes admises à la Clinique de Bel-Air connaît une augmentation de 311\% pour doubler encore entre 1964 et 1970, et cela en raison d'un accroissement démographique de l'ensemble de la population de $44 \%$ entre 1937 et 1960 et de $30 \%$ pour la période allant de 1960 à $1970 .{ }^{102}$ D’après le Dr. Gaston Garrone, médecin-chef du CPSU (le secteur extrahospitalier) en 1973, les traitements de type ambulatoires et la politique extrahospitalière développés sur le territoire genevois ont contribué à l'augmentation de la consommation psychiatrique dans la population, n'engendrant certes pas d'améliorations en termes de traitement des psychoses aiguës, mais diminuant le nombre d'hospitalisation au profit d'une prise en charge ambulatoire qui concerne alors dans son ensemble quelque 6000 personnes. ${ }^{103}$ En 1977, cela concerne 7000 individus, ce qui signifie qu'environ un tiers de la population urbaine se trouve concernée par une intervention d'ordre psychopathologique au moins une fois dans sa vie. ${ }^{104}$ Absente jusqu'aux années $1950,{ }^{105}$ la psychiatrie privée connaît également

100 INFO-BEL-AIR, VPOD, n² 2 , Janvier 1979.

101 Réseau suisse-romand «alternative à la psychiatrie». Trieste, la fin d'un hôpital psychiatrique! Genève, novembre, 1977, bulletin n ${ }^{\circ} 4,40-41$.

102 Johanes-Smidof 1984.

103 Tout Va Bien. LE PREAU DES FOUS. Eté 73.

104 Journal de Genève 1977, 6 décembre.

105 Johanes-Smidof 1984. 
une expansion, parallèlement au développement du secteur public, en raison du fait que l'institution forme davantage de praticiens, ${ }^{106}$ mais aussi en raison de la souffrance manifestée par une partie de la population en quête de soulagement. ${ }^{107}$ À ce propos, le directeur Tissot affirme:

C'est vrai que si l'on ne fait pas attention, on pourrait finir par «psychiatriser» toute la ville. Mais nous avons conscience de ce danger-là, et notre but n'est nullement de chercher à encadrer ou régenter la société, mais d'apporter de l'aide lorsque le besoin en fait ressentir. ${ }^{108}$

Or, à Genève, jusqu'à la fin des années 1970, l'étendue du pouvoir psychiatrique se trouve articulé et légitimé par la loi sur les personnes atteintes d'affection mentale de $1936 .{ }^{109} \mathrm{Si}$ les troubles psychiques sont du domaine de la psychiatrie, le diagnostic, différemment de ce qu'il en est dans les autres branches de la médecine, dépasse largement le domaine médical. À ce propos, Ajuriaguerra souligne que la notion d'aliénation mentale telle qu'elle est définie par la loi de 1936 «nuit considérablement au traitement des malades mentaux et compromet leur réadaptation et réinsertion sociales». ${ }^{110} \mathrm{De}$ ce fait, comme le relève Johanes-Smidof, il semblerait que la société délègue à l'institution psychiatrique la gestion de toute une partie assez hétérogène de la population aux comportements non-conformes, dans le but de la soigner, mais aussi de la contrôler. ${ }^{111}$ Cette allégation est contestée par Tissot. Tout en reconnaissant l'existence potentielle d'abus de la part du pouvoir politique en jouant sur cette difficulté d'établir la limite entre le «normal» et le «pathologique», le directeur de la Clinique souligne que «le devoir des psychiatres et des hôpitaux est de soigner des gens malades et en aucun cas de rééduquer ou transformer des contestataires qui ne sont pas malades». Et il ajoute à ce propos que c'est une «nécessité absolue pour les psychiatres de garder la confiance de la population». ${ }^{112}$

La psychiatrisation de la population, et en particulier les mesures d'internement administratif, font l'objet de réflexion de la part de plusieurs mouvements de la scène alternative en matière de psychiatrie. Au mois de décembre

106 Bovier 1981.

107 TEXTE DU GROUPE «PSYCHIATRIE».

108 Journal de Genève 1977, 27 septembre.

109 L'article 1 de la loi du 1936 sur le régime des personnes atteintes d'affection mentale se décline ainsi:

Sont soumis aux dispositions de la présente loi les personnes résidants dans le canton de Genève et atteintes de maladies mentales et, d'une manière générale, tous les autres malades dont l'état mental est de nature à compromettre la sécurité, la décence ou la tranquillité publique ou leur propre sécurité (LOI sur le régime des personnes atteintes d'affections mentales du 14 mars 1936, 1.)

110 Ajuriaguerra cité par Bovier 1981, 112.

111 Johanes-Smidof 1984.

112 Journal de Genève 1977, 24 septembre, 20. 
1975, l'Association Genevoise des Médecins Progressistes (AMP-Genève) voit le jour; en 1976 naît le Comité contre la répression psychiatrique. Ils prennent part aux réflexions sur la psychiatrie et sur les formes de répression psychiatrique. ${ }^{113}$ En effet, les membres du Comité contre la répression psychiatrique considèrent l'internement administratif ou forcé comme «la clé de voûte de toute l'activité psychiatrique». ${ }^{14}$ Le 26 mai 1976, l'AMPGenève donne naissance au Groupe psychiatrie s'intéressant aux questionnements découlant de l'internement administratif, notamment en termes de privation des droits. ${ }^{115}$ Finalement, face aux problèmes qui continuent d'affecter la psychiatrie genevoise et par conséquent sa population, un groupe de patients participant à une réunion du Réseau suisse-romand d'Alternative à la Psychiatrie exprime la nécessité de créer une association de défense des personnes ayant recours à la psychiatrie, un collectif qui devrait réunir les professionnels de la psychiatrie, les patients, les familles, les juristes, etc. ${ }^{116}$ C'est ainsi qu'en 1979, l'assemblée constitutive donne naissance à Genève à l'ADUPSY (Association pour les droits des usagers de la psychiatrie), avec la volonté d'établir une relation contractuelle en psychiatrie rejoignant ainsi les autres branches de la médecine. ${ }^{117}$ Voici ses principaux objectifs:

\begin{abstract}
Dénoncer, chaque fois qu'il sera possible, l'irresponsabilité civile, considérée comme une sanction. Et repousser sans cesse la frontière responsabilité-irresponsabilité, afin de réduire le nombre des usagers la psychiatrie privés de l'exercice de leurs droits; l'étude critique de tous les projets de révision de la loi sur l'internement et la toxicomanie, dans le souci de préserver et d'augmenter les droits légaux des usagers de la psychiatrie; tenter d'établir des rapports avec les syndicats et des régies pour empêcher toute discrimination à l'égard des usagers de la psychiatrie; enfin, favoriser toute démarche allant dans le sens d'une légitime défense des droits des usagers de la psychiatrie. ${ }^{118}$
\end{abstract}

Cette prise de position semble cependant se confronter aux limites de la perception de la réalité lors des phases aigües et au risque potentiel envers soi-même ou envers de tierces personnes. Tissot souligne à ce propos l'impossibilité d'une relation de type contractuelle lorsque le patient est en phase

113 ASSOCIATION GENEVOISE DE MEDECINS PROGRESSISTES. Genève, le 18.12.75; Réseau Suisse-Romand d'Alternative à la psychiatrie. Sur le développement de la psychiatrie à Genève et au delà de celle-ci.

114 Réseau Suisse-Romand d'Alternative à la psychiatrie. Sur le développement de la psychiatrie à Genève et au delà de celle-ci, 15.

115 ASSOCIATION DE MEDECINS PROGRESSISTES-GENEVE. Compte-rendu de l'Assemblée générale du 14.5.76. Genève, le 24.5.76.

116 La lutte contre les abus de la psychiatrie. Entretien avec M. Alain Riesen.

117 ADUPSY, Association pour les droits des usagers de la psychiatrie.

118 POURQUOI UNE ASSOCIATION POUR LES DROITS DES USAGERS DE LA PSYCHIATRIE? (ADUPSY), 1. 
de délire, alors que dans la majorité des cas, ce contrat peut être rétablit dans de brefs délais:

Car aujourd'hui, même si nous ne sommes pas entièrement maîtres de la maladie mentale, nous obtenons par nos traitements, psychothérapiques, institutionnels, chimiothérapiques et biologiques, des résultats dont le premier signe est justement une prise de conscience de la pathologie. C'est à ce moment-là que le contrat se rétablit. ${ }^{119}$

Or, si, d'une part, la psychiatrie essaye de protéger l'individu malade, d'autre part, elle se rend responsable, par le biais du diagnostic, d'une stigmatisation de l'individu aux conséquences extrêmement onéreuses en termes de participation sociale. Ceci semble dépendre de la conception des troubles psychiques - en particulier de leurs causes - qui se traduit également dans la loi et influence directement le regard qu'une société porte sur ce phénomène. À ce sujet, une fois la personne étiquetée de malade mental, elle se trouve confrontée à toutes sortes de difficultés en matière d'emploi et de logement, jusqu'à l'irresponsabilité civique et l'exclusion par le biais de l'internement, dénonce l'ADUPSY. ${ }^{120}$ Ces répercussions semblent être directement en lien avec une conception médicale de la maladie mentale. D’après Bierens de Haan, la seule explication médicale des troubles psychiques, ou du moins sa prédominance, s'obstine à rechercher l'origine des problèmes au sein de l'individu, minimisant les variables contextuelles et micro-conflictuelles. Ceci transforme la personne qui souffre en objet malade, ${ }^{121}$ la psychiatrie étant ainsi responsable de transformer des problèmes liés à l'existence, en maladies et en symptômes. ${ }^{122}$

Cette vision de la psychiatrie telle qu'elle serait pratiquée dans la Clinique de Bel-Air est contestée par Tissot qui reconnaît le rôle joué par les variables contextuelles dans le déclenchement de la maladie mentale, tout en affirmant l'importance des facteurs génétiques. ${ }^{123}$ Cependant trois jours après, lors de l'interview du 27 septembre 1977, Tissot semble se contredire et souligne la prédominance d'une approche biologique en minimisant les facteurs sociaux qui seraient liés seulement à certaines catégories de maladies à caractère typiquement social. Il affirme en effet:

119 Journal de Genève 1977, 24 septembre, 20.

120 Pour une ASSOCIATION POUR LES DROITS DES USAGERS DE LA PSYCHIATRIE.

121 La communauté thérapeutique dans l'hôpital psychiatrique, courant d'espoir pour soignés et soignants. B. Bierens de Haan.

122 Dr. Bierens de Haan interviewé par De Senarclens 1977, 23 septembre.

123 Journal de Genève 1977, 24 septembre. 
Et là on débouche sur cette aberration de l'antipsychiatrie qui ne voit plus dans la maladie mentale qu'un sous-produit d'une société mal-organisée. Alors que l'on sait parfaitement que - même s'il y a des maladies de société, comme la drogue ou l'alcoolisme - un très grand nombre d'autres correspondent à des troubles biologiques précis.

Et il ajoute:

Lorsqu'un patient a sa liberté subjuguée par des comportements qui deviennent répétitifs, le psychiatre n'a pas à se poser des questions relatives à la «normalité» ou l'«anormalité». Il n'a pas non plus à se poser la question de savoir s'il vaut mieux réformer la société, plutôt que de soigner le malade. ${ }^{124}$

Bien que le but de la psychiatrie soit le bien-être du patient d'un point de vue médical, cela soulève tout de même la question du lien étroit entre l'anomalie des comportements, les normes sociales, la psychiatrie et son rôle au sein d'une société. Or, si Tissot insiste sur la prépondérance des variables médicales, l'ancien directeur de la Clinique Ajuriaguerra reconnaît cette interdépendance et affirme que les problèmes fondamentaux de la psychiatrie sont la liberté et la tolérance, celle du psychiatre tout comme celle de la part de la société. ${ }^{125}$ En effet, dans la majorité des cas, le premier acte de la «carrière psychiatrique» du futur malade relève d'une plainte émanant de son environnement qui révèle l'anomalie de ses comportements. ${ }^{126}$ En Suisse, cette anomalie comportementale n'est cependant pas suffisante en elle-même pour produire une hospitalisation: il faut qu'il y ait un danger potentiel pour le patient lui-même ou pour autrui, affirme Tissot. ${ }^{127} \mathrm{Ce}$ dernier critère retient également notre attention et met ultérieurement en évidence les dimensions politiques et sociales de la psychiatrie, car la dangerosité potentielle n'est ni un facteur strictement médical ni un facteur strictement juridique. De ce fait, la limite entre l'ordinaire et l'anomal est le résultat complexe d'une procédure à caractère arbitraire qui comprend un ensemble de plusieurs variables, relié à plusieurs domaines. À ce sujet les membres de l'ADUPSY relèvent que

si la société opère un choix entre les individus qu'elle accepte et ceux qu'elle n'accepte pas - on peut parler ici de normalité d'un côté et de déviance et délinquance de l'autre, elle le fait par le biais d'organes désignés pour ratifier ses jugements, d'une façon tout à fait claire pour la délinquance, d'une façon plus floue pour la déviance et la folie.128

124 Journal de Genève 1977, 27 septembre, 20.

125 De Ajuriaguerra 1976; Henri Villy 1976, 28 juillet.

126 Bierens de Haan 1979; Goffman 1963/2009.

127 Journal de Genève 1977, 24 septembre.

128 Ibid., 1. 


\section{Débats sur l'internement non volontaire}

La loi de 1936 préconise une psychiatrie de type extrahospitalier reléguant le recours à l'internement comme dernière instance. Le Dr. Garrone relève qu'en 1973, l'internement administratif est en diminution constante en faveur d'une augmentation des admissions volontaires, transformant le régime de Bel-Air de régime d'internement en régime d'hospitalisation. ${ }^{129}$ Cependant, les membres de l'ADUPSY soulignent qu'«en 1975, la majorité des malades mentaux entrent encore à Bel-Air contre leur gré, internés d'urgence». ${ }^{130}$ En effet, en 1975 les admissions libres concernent le $41.92 \%$ des cas. ${ }^{131}$

L'ADUPSY attribue au recours aux mesures d'internement administratif la valeur d'indice manifeste du niveau de tolérance de la société face aux comportements non conformes aux normes, ${ }^{132}$ et prend position «contre toute forme de contrainte en psychiatrie. De principe, elle est en faveur de la suppression complète des internements, ce qui devrait aussi conduire à une modification radicale de la psychiatrie». ${ }^{133}$

Or, empêcher de possibles abus en matière d'internement est la mission du Conseil de Surveillance Psychiatrique (CSP) qui est chargé de garantir le respect de la loi et qui œuvre par conséquent pour la défense des droits des usagers de la psychiatrie. ${ }^{134}$ La réalité parait par contre toute autre, comme le relèvent les membres de l'ADUPSY. En effet, le CSP semble avaliser les internements, volontaires ou non, sur la simple présentation d'un certificat médical. ${ }^{135}$ De plus, le recours à la procédure d'internement d'urgence, qui d'après la loi devrait être l'exception au profit de l'internement non urgent ou de l'admission demandée, donne au médecin le pouvoir d'ordonner un internement à des fins thérapeutiques en seulement quelques minutes, contournant ainsi l'autorisation du Département qui doit être livrée dans les 24 heures. ${ }^{136}$ L'ADUPSY souligne qu'une fois la personne diagnostiquée malade, elle perd son pouvoir contractuel: désormais, pour le patient, sortir

129 Tout Va Bien. LE PREAU DES FOUS. Eté 73.

130 L'association pour les droits des usagers de la psychiatrie (ADUPSY) face à la révision de la loi des personnes atteintes d'affections mentales du 14 mars 1936. Genève, septembre $1979,7$.

131 Henri Villy 1976, 28 juillet.

132 Réseau romand d'alternative à la psychiatrie (Tiré du Rebrousse-Poil: No 2 mars 1978).

133 L'association pour les droits des usagers de la psychiatrie (ADUPSY) face à la révision de la loi des personnes atteintes d'affections mentales du 14 mars 1936. Genève, septembre $1979,9$.

134 LOI sur le régime des personnes atteintes d'affections mentales du 14 mars 1936, Titre II: surveillance des malades.

135 L'association pour les droits des usagers de la psychiatrie (ADUPSY) face à la révision de la loi des personnes atteintes d'affections mentales du 14 mars 1936. Genève, septembre 1979.

136 LOI sur le régime des personnes atteintes d'affections mentales du 14 mars 1936, art. 21. 
de l'hôpital, même s'il y est entré à sa demande, n'est plus une simple formalité. En effet, c'est le médecin qui décide s'il peut quitter la structure. En cas de refus de la part du médecin, le CSP est chargé de vérifier son état, mais dans un laps de temps de deux à cinq jours, délai pendant lequel la personne reste à la merci de la volonté du médecin. ${ }^{137}$ En outre, toute autorisation de sortie est à caractère provisoire et cela pendant la durée d'un mois. ${ }^{138}$ Comme le souligne le Dr. Bierens de Haan: «rien de plus facile que d'entrer dans un hôpital psychiatrique. Les portes s'ouvrent toujours. Il suffit d'attendre. En sens inverse, le trajet est bien plus compliqué et semé d'embûches». ${ }^{139}$ Le directeur Tissot n'est cependant pas du même avis et souligne qu'à Bel-Air, «les patients qui font une entrée contre leur volonté sont, dans un délai d'une dizaine de jours, en pavillon ouvert et demeurent le temps qu'il faut, cela en parfait accord avec nous». ${ }^{140}$ Un autre danger lors de l'internement, tout comme pour la sortie ou la durée du séjour, concerne la frontière entre les symptômes et les réactions à l'enfermement, entre la soumission et l'insoumission au vouloir du personnel soignant. À ce propos, Tissot reconnaît:

Ce danger de voir le personnel soignant céder à la tentation de la routine, donner par exemple plus de médicaments pour avoir la paix [...], nous en sommes très conscients et nous avons à cœur de le prévenir. Tous nos efforts ont pour but de faire du malade mental un malade comme les autres, traité avec les mêmes égards, considéré de la même manière. Mais il y a des affections où la contrainte, voire un rapport de force entre soignant et malade sont inévitables. ${ }^{141}$

Les membres du Réseau suisse-romand d'Alternative à la Psychiatrie dénoncent les critères conduisant au recours à l'internement administratif qui sont de nature très floue et fortement arbitraires dans leurs appréciation: la dangerosité pour autrui ou soi-même, la maladie mentale, des problèmes d'ordre public, etc.; c'est pour cela que des travailleurs du domaine de la psychiatrie et une partie de la population se mobilisent contre cette mesure. ${ }^{142}$ D'après l'ADUPSY, la psychiatrie se caractérise ainsi par l'arbitraire de son statut: une tension donnant lieu à une grande ambiguité où les limites de l'action thérapeutique se confondent avec les dimensions de normalisation

137 L'association pour les droits des usagers de la psychiatrie (ADUPSY) face à la révision de la loi des personnes atteintes d'affections mentales du 14 mars 1936. Genève, septembre 1979.

138 LOI sur le régime des personnes atteintes d'affections mentales du 14 mars 1936, art. 30.

139 Bierens de Haan 1979, 21.

140 Journal de Genève 1977, 24 septembre, 20.

141 Arsever 1979, 10 septembre, 5.

142 Réseau romand d'alternative à la psychiatrie (Tiré du Rebrousse-Poil: No 2 mars 1978). 
et d'exclusion. ${ }^{143}$ La loi de 1936, et en particulier la question de l'internement psychiatrique, occupent une place très importante dans le débat autour de la psychiatrie genevoise. Outre l'affaire de d'Anna B. qui date de 1977, la nature répressive de la psychiatrie et le recours à cette discipline pour résoudre des problèmes d'ordre politique ou social se dévoilent encore publiquement lors de deux affaires d'internement abusif qui se déroulent en janvier 1976: la tentative d'internement forcé d'une commerçante, Madame B., qui ne voulait pas capituler face à l'ordre d'expulsion de son commerce, et l'internement de l'étudiant D., qui contestait la hiérarchie universitaire (internement de la durée d'un mois avec recours à une cure de sommeil contre sa volonté et celle de sa famille). ${ }^{144}$ De la sorte, c'est sur une vague médiatique qu'en 1977, deux projets de modification de loi sont mis en discussion, l'un du parti libéral, l'autre du parti socialiste, tous les deux critiqués par l'ADUPSY:

L'Association pour les droits des usagers de la psychiatrie estime que le débat doit être placé au niveau des Droits de l'homme, afin que les malades mentaux ne voient ni leurs droits ni leurs libertés restreints par le seul fait qu'ils sont malades. ${ }^{145}$

Dans ce sens, d'après l'ADUPSY, la nouvelle loi devrait limiter l'étendue du pouvoir psychiatrique en termes d'internement involontaire, promouvant une psychiatrie fondée sur une base contractuelle, travaillant également à limiter le poids du stigmate découlant de l'étiquette de «malade mental». ${ }^{146} \mathrm{Or}$, ces projets de modification s'inscrivent également dans un contexte fédéral. En effet, lors de la ratification de la Convention européenne des droits de l'homme (CEDH) en 1974, la Suisse a dû formuler une réserve concernant l'article 5 et les conditions qui régissent la privation de liberté. Ainsi, en 1977, afin de se conformer aux dispositions de la CEDH, le Conseil fédéral propose une modification du Code civil suisse en termes de privation de liberté à des fins d'assistance. ${ }^{147}$ La nouvelle loi cantonale devra donc tenir compte de ces modifications du Code civil suisse adoptées en 1978. ${ }^{148}$

Finalement, l'intervention publique de l'ADUPSY n'est pas prise en considération; ${ }^{149}$ la nouvelle loi (K 125 ) adoptée le 7 décembre 1979 relève d'une

143 Pour une ASSOCIATION POUR LES DROITS DES USAGERS DE LA PSYCHIATRIE.

144 Brochure sur la psychiatrie répressive: RAPPEL DE DEUX CAS.

145 L'association pour les droits des usagers de la psychiatrie (ADUPSY) face à la révision de la loi des personnes atteintes d'affections mentales du 14 mars 1936. Genève, septembre 1979,7 .

146 Ibid.

147 Feuille Fédérale. Berne, 26 septembre 1977.

148 L'association pour les droits des usagers de la psychiatrie (ADUPSY) face à la révision de la loi des personnes atteintes d'affections mentales du 14 mars 1936. Genève, septembre 1979.

149 La psychiatrie à Genève. Une crise ou une remise en question plus fondamentale? Mars 1982. 
nature hybride: elle reproduit la structure de la loi de 1936 tout en présentant des modifications issues des deux projets; la commission parlementaire ayant reconnu, d'une part, la volonté de renforcer les droits des personnes ayant recours à la psychiatrie, d'autre part, la nécessité de protéger la société de ces derniers. ${ }^{150}$ Mais les droits des usagers de la psychiatrie restent ainsi toujours insuffisants aux yeux de l'ADUPSY..$^{151}$

\section{Médiatisation et crise ouverte de la psychiatrie}

Les critiques intra et extrahospitalières traversent désormais l'institution psychiatrique: un clivage amplifié par les tensions internes, aggravé par la médiatisation d'autres affaires d'internement arbitraire en 1978 et en 1980, mais surtout exaspéré par le décès de deux patients en 1980. ${ }^{152} \mathrm{Au}$ mois de janvier, un patient meurt d'une atteinte imprévisible du cœur qui, d'après le directeur Tissot, se produit rarement chez des individus sous traitement neuroleptique depuis longtemps. En juin, Alain U., membre de l'ADUPSY, expire lors d'une cure de sommeil qui lui avait été imposée. ${ }^{153}$ D'après l'ADUPSY, alors que le recours aux neuroleptiques est très fréquent dans le traitement des psychoses, la cure de sommeil est désuète et abandonnée presque partout depuis une quinzaine d'années en raison des risques et de la nécessité d'un encadrement infirmier conséquent. ${ }^{154} \mathrm{Ce}$ terme peut cependant prêter à confusion. Sur ce sujet, une dizaine de médecins responsables d'établissement psychiatriques de Suisse romande, désireux d'informer et de rassurer les malades et les familles, expliquent que «le terme scure de sommeil> a survécu alors qu'en fait il ne s'agit plus du tout des cures telles qu'elles se pratiquaient avant l'apparition des médicaments psychotropes». ${ }^{155}$ Ils ajoutent que, en psychiatrie, comme dans toute branche de la médecine, le risque d'accident en cours de traitement est toujours présent. Mais celui d'Alain semble ne pas être un cas isolé. En effet, d'après l'ADUPSY, d'autres morts suspectes ont été dénoncées par le personnel soignant et les familles auprès de l'association. ${ }^{156}$ La Clinique de Bel-Air se trouve ainsi au cœur de

150 Guillod/Hänni 2001.

151 VO GENEVOISE. Internements psychiatriques. Les droits des usagers restent très insuffisants. 28 février 1980.

152 La psychiatrie à Genève. Une crise ou une remise en question plus fondamentale? Mars 1982.

153 Ibid.

154 Communiqué de presse ADUPSY.

155 Guggisberg/de Meuron/Müller/Rémy/Rey-Bellet/Van/van Leckwyck/Schneider/Winteler 1980, 25 juillet. 12.

156 La psychiatrie à Genève. Une crise ou une remise en question plus fondamentale? Mars 1982. 
critiques venant de l'opinion publique, des milieux hospitaliers et du Conseil d'État. ${ }^{157} \mathrm{Ce}$ dernier ordonne la création d'une Commission d'enquête sur les institutions psychiatriques genevoises au mois de septembre 1980: la crise éclate au grand jour. ${ }^{158}$

Riesen, membre de l'ADUPSY, souligne que la particularité du questionnement de ces dernières années, qui le distingue des autres questionnements ayant accompagné de tout temps la discipline psychiatrique dans les Etats démocratiques, est la rupture avec celle qu'il définit comme la «crise normalisée» qui se résolvait avec une suite de réformes. ${ }^{159} \mathrm{Ce}$ nouveau phénomène comporte l'implication et la prise de parole de nombreux groupes dans la population qui interrogent et agissent dans leur propre cadre de vie; un questionnement qui concerne certes la psychiatrie, mais qui dépasse les seules pratiques institutionnelles pour s'attaquer également à leurs implications sociales et politiques. De ce fait, poursuit Riesen, l'ambiguité de l'internement psychiatrique, l'isolement en cellule, le choix et les procédures concernant les traitements psychopharmacologiques, ainsi que les conséquences découlant du stigmate lié au diagnostic, semblent jouer un rôle de catalyseur dans un conflit dont les racines sont profondes. ${ }^{160}$ Cette médiatisation des problèmes internes à la psychiatrie met à jour un ensemble de conflits qui existent depuis bien avant 1976, comme le souligne le groupe VPOD/Bel-Air. ${ }^{161}$

En septembre 1981, la Commission d'enquête dépose son rapport, exposant ouvertement les carences et les contradictions qui caractérisent les institutions psychiatriques de Genève; c'est la première fois que les pouvoirs politiques reconnaissent publiquement l'existence d'une situation problématique. ${ }^{162}$ D'après le rapport d'enquête, suite au départ du directeur Ajuriaguerra (1976) et à la bipolarisation de la direction de la psychiatrie genevoise entre hospitalier et extrahospitalier, on assiste à une période de «désectorisation» (1977-1980) et à l'accroissement de l'écart entre les services hospitaliers et extrahospitaliers. ${ }^{163}$ Une telle régression renforce le rôle joué par la prise en charge hospitalière et le pouvoir du directeur médical de la Clinique favorisant un éventail très restreint en termes de thérapies, essentiellement

157 Bimpage 1981, 26 octobre.

158 Voir Steinauer 1982; Heimberg 2005.

159 La naissance de l'institution pour «aliénés» à Genève au XIX ${ }^{\mathrm{e}}$ siècle et la problématique de l'internement. Par Alain Riesen.

160 Ibid.

161 INFO-BEL-AIR, VPOD, nº 2, Janvier 1979.

162 La psychiatrie à Genève. Une crise ou une remise en question plus fondamentale? Mars 1982.

163 Rapport de la commission d'enquête sur les institutions universitaires psychiatriques genevoises, du $1^{\text {er }}$ septembre 1981. Séance du 8 octobre 1981 (après-midi). 
psychopharmacologique, au détriment d'une approche pluridimensionnelle caractérisant les services extrahospitaliers. En outre, si les membres de la commission d'enquête s'expriment en faveur de l'impossibilité de l'abandon des mesures d'isolement en raison de l'état actuel des connaissances en matière de psychiatrie, ils soulignent qu'à la Clinique de Bel-Air, le recours à cette pratique est très fréquent lors des admissions, parfois dans un but punitif, et que l'état des locaux est inquiétant:164

Nous avons été frappés par l'aspect carcéral des locaux d'isolement (graffiti, déprédations) ainsi que par l'absence de sonnette d'appel dans ces cellules pour deux des trois services d'admission. Il n'existe pas de directives claires quant à la prescription des mesures d'isolement. Celles-ci sont prises en vue de la protection des malades par rapport à eux-mêmes, aux autres patients et au personnel, et aussi pour une sorte de mise en condition face à une phase d'agitation. ${ }^{165}$

Ces constats ne sont pas les seuls qui motivent le Conseil d'État à décréter une réorganisation des institutions psychiatriques genevoises qui se traduit par la suppression des directions médicales hospitalières et extrahospitalières ${ }^{166}$ mais aussi par la création de nouveaux services, notamment celui de recherche en biologie et pharmacologie sous la gestion du Dr. Tissot. D'après le groupe VPOD, ce choix ne fait que consolider le secteur hospitalier au détriment des solutions ambulatoires. ${ }^{167}$ Or, la recherche, depuis longtemps source de débats, sera assujettie au contrôle du Conseil de surveillance psychiatrique et de la Commission d'éthique, ${ }^{168}$ alors que l'ADUPSY dénonce le peu de clarté en termes d'admission et surtout l'exposition des patients à l'arbitraire du pouvoir du médecin. ${ }^{169}$ Dans cette atmosphère de transformations, la VPOD Bel-Air revient à la charge avec des revendications qui datent déjà des années 1970, notamment le développement de la psychiatrie ambulatoire, le droit pour tous à une formation, la diversité des soins ainsi qu'une gestion plus démocratique des structures. ${ }^{170}$

En termes de législation, la nouvelle loi (K 125), adoptée le 7 décembre

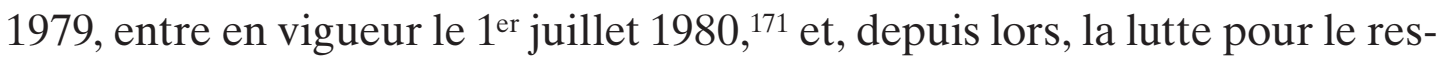
pect des droits des usagers de la psychiatrie se poursuit sur le terrain juridique. En 1982, convaincus de la nécessité de renforcer et défendre les droits

164 Ibid.

165 Ibid., 3755.

166 La psychiatrie à Genève. Une crise ou une remise en question plus fondamentale? Mars 1982.

167 Bimpage 1982, 30 mars.

168 Bimpage 1982, 26 mars.

169 La psychiatrie à Genève. Une crise ou une remise en question plus fondamentale? Mars 1982.

170 Bondi/Heimberg 1981, 18 septembre.

171 Guillod/Hänni 2001. 
des malades, l'ADUPSY et l'AMP proposent de lancer une initiative, acceptée et soutenue par l'ensemble des forces progressistes. Cette initiative se présente comme une conséquence directe des faits survenus à la Clinique de Bel-Air en 1980-1981. ${ }^{172}$ L'initiative, déposée le 13 janvier 1983, ${ }^{173}$ s'articule notamment autour du droit à l'information, au consentement ou non consentement lors d'activités thérapeutiques, médicales ou de recherche. Dans le cas d'une incapacité de discernement le consentement préalable du représentant légal ou d'un membre de la famille est nécessaire. ${ }^{174}$ Soutenue par 17 associations et organisations politiques, cette initiative sera à l'origine de la réforme de la loi K 125 du 7 mai 1987, engendrant des modifications concernant la sortie sur demande du patient, l'interdiction de mise en cellule et des limitations en matière de psychochirurgie. De plus, elle donnera lieu à la création d'une loi ad hoc (loi K 1 80), le 6 décembre 1987. ${ }^{175}$ L'initiative conduira ensuite au vote populaire et à une nouvelle modification de la loi K 125 le 17 juin 1988 qui portera à la suppression de l'alinéa 3 de l'article 23 qui concerne la possibilité de refuser la sortie d'un patient suite à une admission volontaire. ${ }^{176}$ Cet alinéa avait déjà fait l'objet d'une première pétition déposée par la VPOD/Groupe Bel-Air et l'Association suisse des infirmiers et infirmières des institutions universitaires de psychiatrie en janvier $1981 .{ }^{177}$

\section{Conclusion}

La critique de l'institution psychiatrique se développe dans le Canton de Genève à partir des années 1970 et est caractérisée par un élément novateur, c'est-à-dire la mobilisation d'une pluralité de groupes et mouvements qui comprennent également les usagers de la psychiatrie eux-mêmes. Cette mobilisation s'inscrit dans un plus ample phénomène international de remise en cause des pouvoir institutionnels, tout comme des normes sociales, qui dépasse le domaine de la psychiatrie. Au niveau genevois, nous pouvons remarquer que les acteurs et actrices de la critique psychiatrique jouent un rôle actif dans la médiatisation des abus de la psychiatrie jusqu'à l'éclatement de

172 L'université ouvrière de Genève organise une table ronde autour de l'initiative sur le «droit des malades», 23 septembre 1982.

173 Comité d'initiative pour les droits des malades. Genève, le 14.2.1983.

174 Comité unitaire pour un tarif plus social de la médecine ambulatoire. Genève, 3 mars 1982.

175 Guillod/Hänni 2001.

176 Ibid.

177 Rapport de la commission des pétitions sur la pétition de la Fédération suisse du personnel des services public/groupe Bel-Air et de l'Association suisse des infirmiers et infirmières des institutions universitaires de psychiatrie. 
la crise et la prise de conscience de la part des autorités des dysfonctionnements de la psychiatrie genevoise. Ce constat a plusieurs conséquences pour l'institution psychiatrique et représente un tournant important en matière de soins des troubles psychiques. Outre la réorganisation de l'institution, cet engagement et la crise y faisant suite ouvrent la voie à plusieurs modifications juridiques, mais force est de constater que tous les espoirs et revendications des groupes et mouvements militant en faveur d'une psychiatrie alternative n'ont pas abouti. Comme le relèvent les membres de l'ADUPSY, les années 1980 marquent le déclin des espérances pour ce qui concerne la fin de l'asile et de la répression psychiatrique. ${ }^{178}$

Dans les années 1980 l'atmosphère contestataire et militante postsoixante-huitarde semble s'essouffler face aux tensions qui caractérisaient et caractérisent toujours le domaine de la psychiatrie. En effet, bien qu'affaiblie, l'approche médicale de la psychiatrie maintient son ascendant face aux formes de souffrance psychique tout en incluant d'autres approches théoriques et thérapeutiques. Ceci pourrait s'expliquer d'une part par une institutionnalisation des formes de contestation et d'autre part par les difficultés d'interprétation et d'évaluation lors des situations de crise, de danger potentiel ou d'incapacité de discernement. En effet, si le renforcement des droits des usagers de la psychiatrie permet à présent une majeure protection et limite les abus en matière de psychiatrie, le pouvoir médical garde son emprise lors de l'expertise. Le désir d'établir une psychiatrie sur une base contractuelle et l'abolition de toute forme de contrainte en psychiatrie ont ainsi devant eux encore beaucoup d'obstacles à franchir.

\section{Bibliographie}

Barham, Peter, «From the Asylum to the Community: the Mental Patient in Postwar Britain», dans: Marijke Gijswijt-Hofstra/Roy Porter (eds.), Cultures of Psychiatry and Mental Health Care in Postwar Britain and the Netherlands (Amsterdam 1998) 221-240

Barnes, Mary/Joseph Berke, Mary Barnes, un voyage à travers la folie (Paris 1973) (Original 1971)

Basaglia, Franco, Che cos'è la psichiatria, in: Franco Basaglia (a cura di), $C H E$ COS'E' LA PSICHIATRIA? (Parme 1967) 15-23

Basaglia, Franco, «Présentation», dans: Franco Basaglia (éd.), L'institution en négation (Paris 2012a) 21-23 (Original 1968)

Basaglia, Franco, «Les institutions de la violence», in: Franco Basaglia (éd.), L'institution en négation (Paris 2012b) 123-159 (Original 1968)

178 L'ADUPSY. Quel avenir? Assemblée générale mercredi 6 juin 20h30, maison de la Jonction, bulletin $\mathrm{n}^{\circ} 2$. 
Basaglia, Franco/Franca Basaglia-Ongaro (a cura di), La maggioranza deviante. L'ideologia del controllo sociale totale (Milan 1971/2013)

Basaglia, Franco, Conferenze Brasiliane (Milan 2000)

Bierens de Haan, Barthold, Dictionnaire critique de la psychiatrie (Lausanne 1979)

Bovier, Philippe, De l'asile à la cité, thèse de doctorat en médecine, Université de Genève (Genève 1981)

Boyers, Robert (éd.), Ronald Laing et l'antipsychiatrie (Paris 1973) (Original 1971)

Brulhart, Armand, Du Mal de Saint-Antoine à Belle-Idée. 2 siècles de psychiatrie à Genève 1800-2000. Tome 1: 1800-1950 (Genève 2002)

Brulhart, Armand, «Essai de chronologie», in: Armand Brulhart (éd.), De Bel-Air à Belle-Idée. 2 siècles de psychiatrie à Genève 1800-2000. Tome 2: 1950-2000 (Genève 2003) 233-348

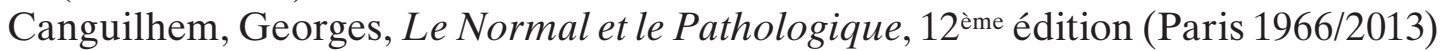

Castel, Robert, La gestion des risques. De l'anti-psychiatrie à l'après-psychanalyse (Paris 1981)

Coffin, Jean-Christophe, «Ronald Laing et l'antipsychiatrie», dans: Geneviève Dreyfus-Armand (éd.), Les années 68 un monde en mouvement. Nouveaux regards sur une histoire plurielle (1962-1981) (Paris, 2008) 332-344

Cooper, David, Psychiatrie et Anti-psychiatrie (Paris 1970) (Original 1967)

Cooper, David, Mort de la famille (Paris 1972) (Original 1971)

Cooper, David, «Lettre au réseau international sur le pouvoir et la différence», dans: Mony Elkaïm (éd.), Réseau-alternative à la psychiatrie. Collectif international (Paris 1977) 35-45

Corbellini, Gilberto/Giovanni Jervis, La razionalità negata. Psichiatria e antipsichiatria in Italia (Turin 2008)

Crossley, Nick, Contesting psychiatry. Social Movements in Mental Health (Londres 2006)

De Ajuriaguerra, Julian, «Enfermement et liberté», dans: Les services psychiatriques de Genève, 1900-1975 (Genève 1976)

Dotti, Federico, La remise en question de la psychiatrie: du mouvement de l'antipsychiatrie aux groupes militants dans la Genève des années 1970, mémoire de maîtrise en sciences de l'éducation, Université de Genève (Genève 2015)

Droze, Jean-Claude, «L'extrahospitalier: une psychiatrie plus sociale?», dans: Armand Brulhart (éd.), De Bel-Air à Belle-Idée. 2 siècles de psychiatrie à Genève 1800-2000. Tome 2 (Genève 2003) 77-81

Elkaïm, Mony, «Présentation», dans: Mony Elkaïm (éd.), Réseau-alternative à la psychiatrie. Collectif international (Paris 1977) 7-11

Fangerau, Heiner/Karen Nolte (Hrsg.), «Moderne» Anstaltspsychiatrie im 19. und 20. Jahrhundert: Legitimation und Kritik (Stuttgart 2006)

Fauvel, Aude, Témoins aliénés et «Bastilles modernes»: une histoire politique, sociale et culturelle des asiles en France (1800-1914), Thèse de doctorat en histoire, Ecole des Hautes Etudes en Sciences Sociales (Paris 2005)

Foot, John, La «repubblica dei matti». Franco Basaglia e la psichiatria radicale in Italia, 1961-1978 (Milan 2014)

Foucault, Michel, Maladie mentale et psychologie, $5^{\mathrm{ème} e}$ édition (Paris 1954/2011)

Foucault, Michel, Histoire de la folie à l'âge classique (Paris 1972/2013) 
Foucault, Michel, «Il faut défendre la société». Cours au Collège de France. 1976 (Paris 1997)

Foucault, Michel, Le Pouvoir psychiatrique. Cours au Collège de France. 1973-1974 (Paris 2003)

Fussinger, Catherine, «Eléments pour une histoire de la communauté thérapeutique dans la psychiatrie occidentale de la seconde moitié du 20e siècle», Gesnerus 67 (2010) 217-240

Galimberti, Umberto, Psichiatria e fenomenologia (Milan 1979/2006)

Gijswijt-Hofstra, Marijke, «Introduction: Cultures of Psychiatry and Mental Health Care in Postwar Britain and the Netherlands», dans: Marijke Gijswijt-Hofstra/ Roy Porter (eds.), Cultures of Psychiatry and Mental Health Care in Postwar Britain and the Netherlands (Amsterdam 1998) 1-7

Goffman, Erving, La mise en scène de la vie quotidienne 2, les relations en publique (Paris 2009) (Original 1963)

Goffman, Erving, Asiles, études sur la condition sociale des malades mentaux (Paris 2013) (Original 1961)

Gros, Dominique, Dissidents du quotidien. La scène alternative genevoise 19681987 (Lausanne 1987)

Gros, Dominique, «du désir de révolution à la dissidence. Constitution de la mouvance alternative genevoise et devenir de ses acteurs», Equinoxe 24 (2004) 31-42

Guattari, Félix La Révolution moléculaire (Paris 2012)

Guillod, Olivier/Hänni, Christian, «Les droits des personnes en psychiatrie. Évaluation juridique», Les cahiers de l'action sociale et de la santé 15 (Genève 2001) 3-64

Heimberg, Charles, «La contestation de l'institution psychiatrique et sa dimension politique: Bel-Air dans l'œil du cyclone (1977-1983)», Cahiers d'histoire du mouvement ouvrier 21 (2005) 187-195

Henckes, Nicolas, Le nouveau monde de la psychiatrie française. Les psychiatres, l'Etat et la réforme des hôpitaux psychiatriques de l'après guerre aux années 1970, thèse de doctorat en sociologie, Ecole des Hautes Etudes en Sciences Sociales (Paris 2007)

Ingleby, David, «The View from the North Sea», dans: Marijke Gijswijt-Hofstra/Roy Porter (eds.), Cultures of Psychiatry and Mental Health Care in Postwar Britain and the Netherlands (Amsterdam 1998) 295-314

Jervis, Giovanni, Le Mythe de l'antipsychiatrie (Paris 1977)

Jervis, Giovanni, «Crise de la psychiatrie et contradictions institutionnelles», dans: Franco Basaglia (éd.), L’institution en négation (Paris 2012) 249-269 (Original 1968)

Joanes-Smidof, Madeleine, Mouvements de la population de la Clinique de Bel-Air, thèse de doctorat en médicine, Université de Genève (Genève 1984)

Jones, Colin, «Raising the Anti: Jan Foudraine, Ronald Laing and Anti-Psychiatry», dans: Marijke Gijswijt-Hofstra/Roy Porter (eds.), Cultures of Psychiatry and Mental Health Care in Postwar Britain and the Netherlands (Amsterdam 1998) 283-294

Laing, Ronald, David, Le Moi divisé (Paris 1970) (Original 1960)

Laing, Ronald, David, Soi et les autres (Paris 1971) (Original 1961)

Laing, Ronald, David, La Politique de la famille (Paris 1979) (Original 1969)

Quétel, Claude, Histoire de la folie, de l'antiquité à nos jours (Paris 2009) 
Rebetez, Niels, Pour une psychiatrie déterritorialisée. Antipsychiatrie dans l'Arc lémanique (1967-1985), mémoire de master en lettres, Université de Fribourg (Fribourg 2014)

Steinauer, Jean. Le Fou du Rhône. Documents sur la crise psychiatrique genevoise (Genève 1982)

Szasz, Thomas, Le Mythe de la maladie mentale (Paris 1975) (Original 1961)

Szasz, Thomas, Fabriquer la folie (Paris 1976) (Original 1970)

Szasz, Thomas, La loi, la liberté et la psychiatrie (Paris 1977) (Original 1963)

Thifault, Marie-Claude/Henri Dorvil (éds.), Désinstitutionnalisation psychiatrique en Acadie, en Ontario francophone et au Québec, 1930-2013 (Québec 2014)

Thomson, Mathew, «Before Anti-Psychiatry: «Mental Health» in Wartime Britain», dans: Marijke Gijswijt-Hofstra/Roy Porter (eds.), Cultures of Psychiatry and Mental Health Care in Postwar Britain and the Netherlands (Amsterdam 1998) 43-59

Von Bueltzingsloewen, Isabelle, L'Hécatombe des fous. La famine dans les hôpitaux psychiatriques français sous l'Occupation (Paris 2007)

Von Bueltzingsloewen, Isabelle, «Quel(s) malade(s) pour quel asile? Le débat sur l'internement psychiatrique dans la France de l'entre-deux-guerres», dans: Laurence Guignard/Hervé Guillemain/Stéphane Tison (éds.), Expériences de la folie. Criminels, soldats, patients en psychiatrie (XIX ${ }^{e}-X X^{e}$ siècles) (Rennes 2013)

\section{Sources}

ADUPSY, Association pour les droits des usagers de la psychiatrie (Archives contestataires, fonds Riesen-Schuler, 005_R-S_S03)

L'ADUPSY. Quel avenir? Assemblée générale mercredi 6 juin 20h30, maison de la Jonction, bulletin no 2 (Archives contestataires, fonds Riesen-Schuler, 005_R-S_ S03)

Communiqué de presse ADUPSY (Archives contestataires, fonds Riesen-Schuler, 005_R-S_S04_SS2)

Arsever, Sylvie, L'internement psychiatrique en question (II). Quel recours contre un internement abusif? Gazette de Lausanne, 5 (1979, 10 septembre $)^{179}$

L'assemblée des médecins-assistants et chefs de clinique de la Clinique Psychiatrique de Bel-Air. Genève, le 4 juin 1974. (Archives contestataires, fonds Riesen-Schuler, 005_R-S_S04_SS3)

ASSOCIATION GENEVOISE DE MEDECINS PROGRESSISTES. Genève, le 18.12.75 (Archives contestataires, fonds AMP, 008_AMP_S01)

ASSOCIATION DE MEDECINS PROGRESSISTES-GENEVE. Compte-rendu de l'Assemblée générale du 14.5.76. Genève, le 24.5.76 (Archives contestataires, fonds AMP, 008_AMP_S01)

POURQUOI UNE ASSOCIATION POUR LES DROITS DES USAGERS DE LA PSYCHIATRIE? (ADUPSY). Genève, décembre 1978 (Archives contestataires, fonds Riesen-Schuler, 005_R-S_S03)

179 Les articles parus dans le Journal de Genève et dans la Gazette de Lausanne cités dans ce travail se trouvent sous forme numérique à l'adresse internet: www.letempsarchives.ch. Lorsque l'article n'est pas signé ou qu'on y trouve seulement les initiales, le «Journal de Genève» ou la «Gazette de Lausanne» est cité comme auteur. 
Pour une ASSOCIATION POUR LES DROITS DES USAGERS DE LA PSYCHIATRIE (Archives contestataires, fonds Riesen-Schuler, 005_R-S_S03)

L'association pour les droits des usagers de la psychiatrie (ADUPSY) face à la révision de la loi des personnes atteintes d'affections mentales du 14 mars 1936. Genève, septembre 1979 (Archives contestataires, fonds Riesen-Schuler, 005_R-S_S04_SS1)

Bimpage, Serge, Bel-Air en question (I). Bel-Air est-il le symptôme d'un mal plus général? Journal de Genève, 17 (1981, 26 octobre)

Bimpage, Serge, Bel-Air en question (II). Vers une psychiatrie d'avant-garde? Journal de Genève, 14 (1981, 27 octobre)

Bimpage, Serge, Bel-Air: les jeux sont faits. Journal de Genève, 19 (1982, 26 mars)

Bimpage, Serge, Réorganisation de la psychiatrie: la VPOD proteste. Journal de Genève, 15 (1982, 30 mars)

Bondi, Michel/Heimberg, Charles, Psychiatrie à Genève la VPOD Bel-Air réagit. Journal de Genève, 25 (1981, 18 septembre)

CAS 1. Journal du Comité d'Action «Santé». Mai 1972. (Archives contestataires, Fonds Jaques Siron, 011_JS_S01_SS1)

Comité d'initiative pour les droits des malades. Genève, le 14.2.1983. (Archives contestataires, fonds Rolf Himmelberger, 027_RH_2)

Comité unitaire pour un tarif plus social de la médecine ambulatoire. Genève, 3 mars 1982. (Archives contestataires, fonds Rolf Himmelberger, 027_RH_2)

La communauté thérapeutique dans l'hôpital psychiatrique, courant d'espoir pour soignés et soignants. B. Bierens de Haan (Archives contestataires, fonds Riesen-Schuler, 005_R-S_S04_SS1)

De Senarclens, Hubert, Psychiatrie à Genève: querelle des anciens et des modernes. «Un défaut de la psychiatrie: avoir transformé des gens avec des problèmes en malades avec des symptômes». Journal de Genève, 21 (1977, 23 septembre)

Feuille Fédérale, 129e année, volume III, N³9. Berne, 26 septembre 1977 (https:// www.amtsdruckschriften.bar.admin.ch/viewOrigDoc.do?ID=10101939)

TEXTE DU GROUPE «PSYCHIATRIE» (Archives contestataires, fonds AMP, 008_AMP_S01)

Guggisberg, Michel/de Meuron, Michel/Müller, Christian/Rémy, Maurice/ReyBellet, Jean/Van, Harutyum/van Leckwyck, Edouard/Schneider, Georges/ Winteler, Ralph, Après un décès en cure de sommeil. Bel-Air: des médecins romands parlent. Journal de Genève, 12 (1980, 25 juillet)

Info-Bel-Air, VPOD, n 2, Janvier 1979 (Archives du Groupe Bel-Air de la VPOD en cours de dépôt)

Journal de Genève, Médecins écartés de Bel-Air: lettre ouverte des syndicats. Journal de Genève, 11 (1977, 28 juillet)

Journal de Genève, Psychiatrie à Genève: querelle des anciens et des modernes (III). Prof. Tissot: «c'est une nécessité absolue pour le psychiatre de garder la confiance de la population». Journal de Genève, 20 (1977, 24 septembre)

Journal de Genève, Psychiatrie à Genève: querelle des anciens et des modernes (IV). «L'antipsychiatrie a tort de ne voir dans la maladie mentale qu'un sous produit d'un monde inorganisé». Journal de Genève, 12 (1977, 27 septembre)

Journal de Genève, Hausse des troubles psychiques dans la population. Journal de Genève, 17 (1977, 6 décembre) 
Julian de Ajuriaguerra Interview lors de l'émission en direct avec du 4 octobre 1971 http://www.rts.ch/archives/tv/information/en-direct-avec/3462537-julian-de-ajuriaguerra.html

Jullien, Odile, Le directeur de la clinique de Bel-Air déplace deux médecins. Journal de Genève, 18 (1977, 25 juin)

LOI sur le régime des personnes atteintes d'affections mentales du 14 mars 1936 (http://www.rolfhimmelberger.ch/wp-content/uploads/2012/03/Loi_K_1_12_ du_36.03.14_Etat-au_71.06.21.pdf)

La lutte contre les abus de la psychiatrie. Entretien avec M. Alain Riesen (http:// edutechwiki.unige.ch/dewiki/Entretien_avec_M._Alain_Riesen) ${ }^{180}$

La naissance de l'institution pour «aliénés» à Genève au XIX ${ }^{\mathrm{e}}$ siècle et la problématique de l'internement. Par Alain Riesen (Archives contestataires, fonds Riesen-Schuler, 005_R-S_S04_SS5)

La psychiatrie à Genève. Une crise ou une remise en question plus fondamentale? Mars 1982. (Archives contestataires, fonds Riesen-Schuler, 005_R-S_ S04_SS5)

Brochure sur la psychiatrie répressive: RAPPEL DE DEUX CAS (Archives contestataires, fonds Rolf Himmelberger, 027_RH_21)

Rapport de la commission d'enquête sur les institutions universitaires psychiatriques genevoises, $d u 1^{\text {er }}$ septembre 1981. Séance du 8 octobre 1981 (aprèsmidi) (http://www.rolfhimmelberger.ch/wp-content/uploads/2012/03/Rapport-comm-enquete-IUPG_81.09.01_Mem-GC_1981_3738-3781.pdf)

Rapport de la commission des pétitions sur la pétition de la Fédération suisse du personnel des services public/groupe Bel-Air et de l'Association suisse des infirmiers et infirmières des institutions universitaires de psychiatrie (http://www.rolfhimmelberger.ch/wp-content/uploads/2012/03/P-465_Rapportcomm-pet_pet-VPOD-ASI_83.11.15.pdf)

Réseau suisse-romand «alternative à la psychiatrie». Genève, mai 1976, bulletin $\mathrm{n}^{\mathrm{o}} 2$ (Archives contestataires, fonds Riesen-Schuler, 005_R-S_S02)

Réseau Suisse-Romand «Alternative à la Psychiatrie», La guerre des psychiatres aura-t-elle lieu? Genève, juillet 1977, bulletin n 3 (Archives contestataires, fonds Riesen-Schuler, 005_R-S_S02)

Réseau suisse-romand «alternative à la psychiatrie». Trieste, la fin d'un hôpital psychiatrique! Genève, novembre, 1977, bulletin n ${ }^{\circ} 4$ (Archives contestataires, fonds Riesen-Schuler, 005_R-S_S02)

Réseau romand d'alternative à la psychiatrie (Tiré du Rebrousse-Poil: No 2 mars 1978). (Archives contestataires, fonds Riesen-Schuler, 005_R-S_S02)

Réseau Suisse-Romand d'Alternative à la psychiatrie. Sur le développement de la psychiatrie à Genève et au delà de celle-ci (Archives contestataires, fonds Rolf Himmelberger, 027_RH_8)

180 Ce témoignage a été recueilli dans le cadre d'un atelier de recherche portant sur les droits de la personne lors du cours Les institutions d'éducation spéciale entre archives, mémoire et histoire, deuxième moitié du $\mathrm{XX}^{e}$ siècle donné par la professeure Martine Ruchat à l'Université de Genève (2013). 
Tout Va Bien. LE PREAU DES FOUS. Eté 73 (Archives contestataires, bibliothèque)

Travail social et Psychiatrie. Séance du lundi 5 novembre 1973. (Archives contestataires, Fonds Jaques Siron, 011_JS_S01_SS1)

L'université ouvrière de Genève organise une table ronde autour de l'initiative sur le «droit des malades», 23 septembre 1982 (Archives contestataires, fonds Rolf Himmelberger, 027_RH_2)

Villy, Henri, La clinique psychiatrique de Bel-Air a 75 ans. Journal de Genève (1976, 28 Juillet)

VO GENEVOISE. Internements psychiatriques. Les droits des usagers restent très insuffisants. 28 février 1980 (Archives contestataires, fonds Rolf Himmelberger, 027_RH_30) 